\title{
STRESS CONSTRAINED G CLOSURE AND RELAXATION OF STRUCTURAL DESIGN PROBLEMS
}

\author{
$\mathrm{BY}$ \\ ROBERT LIPTON \\ Department of Mathematics, Louisiana State University, Baton Rouge, LA 70803
}

\begin{abstract}
A generic relaxation for stress constrained optimal design problems is presented. It is accomplished by introducing the stress constrained $\mathrm{G}$ closure. For a finite number of stress constraints, an explicit characterization of the stress constrained $\mathrm{G}$ closure is given. It is shown that the stress constrained $\mathrm{G}$ closure is characterized by all $\mathrm{G}$ limits together with their derivatives. A local representation of the set of all $\mathrm{G}$ limits and their derivatives is developed.
\end{abstract}

1. Introduction. In the absence of stress constraints, a large body of theory has been developed that facilitates the numerical solution of structural design problems for multiphase solid structures. References to the literature and overviews of the subject can be found in [1], [6], [8], [16], and [29]. In addition to designing for structural response, it is also of central importance to control the stress inside structural components made from composite materials. Regions of large stresses are most likely to be the first to exhibit failure during service. The objective of this work is to identify the relaxed design problem for stress constrained design.

We consider structural elements made from $\mathrm{N}$ linearly elastic materials with elasticity tensors $\mathbf{A}_{1}, \mathbf{A}_{2}, \mathbf{A}_{3}, \ldots, \mathbf{A}_{N}$. The structural domain is a bounded set $\Omega$ in $\mathbf{R}^{3}$ with Lipschitz continuous boundary. A generic point in $\Omega$ is denoted by $\mathbf{x}$. The elastic phases occupy the $N$ subsets $\Omega_{1}, \Omega_{2}, \ldots, \Omega_{N}$ where $\Omega_{1} \cup \Omega_{2} \cup \cdots \cup \Omega_{N}=\Omega$. A particular choice of component elasticity tensors is specified by the array $\underline{\mathbf{A}}=\left(\mathbf{A}_{1}, \mathbf{A}_{2}, \mathbf{A}_{3}, \ldots, \mathbf{A}_{N}\right)$. The local elasticity tensor $\mathbf{C}(\underline{\mathbf{A}}, \mathbf{x})$ is piecewise constant and takes the value $\mathbf{C}(\underline{\mathbf{A}}, \mathbf{x})=\mathbf{A}_{i}$ in the $i$ th material. Denoting the indicator function of the $i$ th material by $\chi_{i}$, the local elasticity tensor is written $\mathbf{C}(\underline{\mathbf{A}}, \mathbf{x})=\sum_{i=1}^{N} \chi_{i}(\mathbf{x}) \mathbf{A}_{i}$. Here $\chi_{i}=1$ in the $i$ th material and zero outside. The design space consists of all partitions of $\Omega$ into Lebesgue measurable subsets $\Omega_{i}, i=1,2, \ldots, N$, occupied by the different materials subject to the resource constraints meas $\left(\Omega_{i}\right) \leq \gamma_{i}$. Here $\sum_{i} \gamma_{i} \geq$ meas $(\Omega)$ and the vector of resource constraints is written $\underline{\gamma}=\left(\gamma_{1}, \gamma_{2}, \ldots, \gamma_{N}\right)$.

Received July 29, 2002.

2000 Mathematics Subject Classification. Primary 74Q05, 74P99. 
For a body load $\mathbf{f}$ in $W^{-1.2}\left(\Omega, \mathbf{R}^{3}\right)$, the elastic displacement $\mathbf{u}$ is the $W_{0}^{1,2}\left(\Omega, \mathbf{R}^{3}\right)$ solution of

$$
-\operatorname{div}(\mathbf{C}(\underline{\mathbf{A}}, \mathbf{x}) \epsilon(\mathbf{u}))=\mathbf{f} .
$$

The stress at each point in the composite $\sigma$ is given by the constitutive relation

$$
\sigma(\mathbf{x})=\mathbf{C}(\underline{\mathbf{A}}, \mathbf{x}) \epsilon(\mathbf{u}(\mathbf{x})) .
$$

Here $\epsilon(\mathbf{u})$ is the strain tensor given by

$$
\epsilon(\mathbf{u})_{i j}=\left(u_{i, j}+u_{j . i}\right) / 2 .
$$

where $u_{i}$ is the $i$ th component of displacement $\mathbf{u}$ and $u_{i . j}$ is its partial derivative in the $j$ th coordinate direction. The equilibrium equation (1.1) holds in the weak sense; i.e., for every $\mathbf{v}$ in $W_{0}^{1,2}\left(\Omega, \mathbf{R}^{3}\right)$.

$$
\int_{\Omega} \mathbf{C}(\underline{\mathbf{A}}, \mathbf{x}) \epsilon(\mathbf{u}): \epsilon(\mathbf{v}) d \mathbf{x}=\int_{\Omega} \mathbf{f} \cdot \mathbf{v} d \mathbf{x} .
$$

The contractions $\mathbf{C}(\underline{\mathbf{A}}, \mathbf{x}) \epsilon(\mathbf{u}): \epsilon(\mathbf{v})$ and $\mathbf{f} \cdot \mathbf{v}$ are given by $\mathbf{C}_{i j k l}(\underline{\mathbf{A}}, \mathbf{x}) \epsilon(\mathbf{u})_{i j} \epsilon(\mathbf{v})_{k l}$ and $\mathbf{f}_{i} v_{i}$ respectively where repeated subscripts indicate summation.

The design criterion is a function of the elastic field $\mathbf{u}$ and is denoted by $F(\mathbf{u})$. The type of structural design criteria considered here are those associated with the compliance of the structure given by $F(\mathbf{u})=\int_{\Omega} \mathbf{f} \cdot \mathbf{u} d \mathbf{x}$ or the distance of the displacement from a desired target displacement $\hat{\mathbf{u}}$ given by $F(\mathbf{u})=\int_{\Omega}|\mathbf{u}-\hat{\mathbf{u}}|^{2}, d \mathbf{x}$. Both of these functions are continuous with respect to weak convergence in $W^{1.2}\left(\Omega, \mathbf{R}^{3}\right)$. However, the results given here are not restricted to this situation and apply to multi-load design problems or any design criteria that is continuous with respect to $G$ convergent sequences of designs [5]. The objective of the design problem is to optimize $F(\mathbf{u})$ over the class of admissible configurations subject to constraints on the stress. One measure of stress concentration is given by the sup-norm of the stress field in the composite body. This is denoted by

$$
\||\sigma|\|_{x}=\operatorname{essisup}|\sigma(\mathbf{x})| \text {. }
$$

where $|\sigma|=\sqrt{\sigma: \sigma}$. We introduce the prototypical design problem given by

$$
P^{\infty}=\inf _{\substack{\text { Configurations. } \\ \text { meas }\left(\Omega, \Omega_{1}\right) \leq \gamma_{i}}} F(\mathbf{u}) .
$$

subject to

$$
\||\sigma|\|_{\infty} \leq K
$$

where $\mathbf{u}$ is a solution of the equation of state (1.1) and $\sigma=\mathbf{C}(\underline{\mathbf{A}}, \mathbf{x}) \epsilon(\mathbf{u})$. Here $K$ is a preset tolerance. We point out that the constraint (1.7) is equivalent to the infinite number of constraints given by

$$
\int_{\Omega} p_{j}(\mathbf{x})|\sigma|^{2} d \mathbf{x} \leq K^{2} \int_{\Omega} p_{j}(\mathbf{x}) d \mathbf{x} . \text { for all } p_{j}(\mathbf{x})
$$

for a countably dense set $\left\{p_{j}\right\}_{j=1}^{\infty}$ of the nonnegative functions in $C^{\infty}(\bar{\Omega})$. From (1.8) it is clear that a theory for design problems with a finite number of stress constraints given by

$$
P^{L}=\inf _{\substack{\text { Configurations. } \\ \text { meas }\left(\$ 2_{i}\right) \leq \gamma_{i}}} F(\mathbf{u}) .
$$


subject to

$$
\int_{\Omega} p_{j}(\mathbf{x})|\sigma|^{2} d \mathbf{x} \leq K^{2} \int_{\Omega} p_{j}(\mathbf{x}) d \mathbf{x}, \text { for } j=1, \ldots, L,
$$

is a prerequisite for understanding (1.6).

The design problems stated above are not readily amenable to numerical solution. The fundamental reason for this is that problems of this type do not possess configurations for which the infimum in (1.6) and (1.9) are attained; see [15], [19], and [26]. Thus any approach that seeks to identify optimal configurations is likely to fail. Instead one sceks to identify minimizing sequences of configurations that approach the infimum in (1.6) and (1.9). Here the objective is to identify nearly optimal configurations. Methods developed for this purpose are known as relaxation methods; see [4] and [10]. Here the original design problem is replaced by a "relaxed version" that is used to identify minimizing sequences of configurations for the original design problem.

The generic feature of minimizing sequences is the appearance of zones in which infinitesimally fine oscillations of material properties occur. This motivates the extension of the design space to the set of all $\mathrm{G}$ limits of sequences of local elasticity tensors associated with configurations of the component materials; see [17] and [20]. Roughly speaking, a $\mathrm{G}$ limit can be thought of as an elasticity tensor that takes values in the set of effective elasticity tensors associated with mixtures of the $\mathrm{N}$ component materials. This extension of the design space suffices to produce the desired relaxed problem for optimizing structural performance in the absence of stress constraints. The extended design space is referred to as the $\mathrm{G}$ closure of the set of local elasticity tensors $\mathbf{C}(\underline{\mathbf{A}}, \mathbf{x})$; see [6].

When dealing with stress constrained problems, a strictly smaller extension of the design space is required. For stress constrained problems, one again extends the space to include $\mathrm{G}$ limits. However, in this context, it is seen that the relaxation is obtained by the extension over the subclass of configurations for which the stress constraints (1.7) or (1.10) are satisfied; see Theorem 4.1. This extension of the design space is referred to as the stress constrained $G$ closure of the set of local elasticity tensors $\mathbf{C}(\underline{\mathbf{A}}, \mathbf{x})$. For a finite number of stress constraints, an explicit characterization of the stress constrained $\mathrm{G}$ closure is given in Theorem 4.2. It is seen that the stress constrained G closure is characterized by all $\mathrm{G}$ limits together with their derivatives. This is used to develop an explicit version of the relaxed stress constrained design problem; see Theorems 4.3 and 4.4. An explicit upper bound for the stress constrained $\mathrm{G}$ closure associated with (1.7) is presented in Theorem 4.5. The homogenized design problem associated with the upper bound is given in Theorem 4.6 and its relation to nearly optimal configurations is given in Theorem 4.7.

In order to characterize the stress constrained $\mathrm{G}$ closure, one is obliged to compute the stress constraints (1.10) for $\mathrm{G}$ converging sequences of tensors. The difficulty stems from the fact that the limit of the stress constraints is not given by the stress constraint applied to the weak limit. This is due to the lack of continuity that can be expressed as follows. Given a sequence of stresses $\left\{\sigma^{n}\right\}_{n=1}^{\infty}$ converging weakly in $L^{2}$ to $\sigma^{M I}$, one has that

$$
\lim _{n \rightarrow \infty} \int_{\Omega} p_{j}(\mathbf{x})\left|\sigma^{n}\right|^{2} d \mathbf{x} \geq \int_{\Omega} p_{j}(\mathbf{x})\left|\sigma^{M I}\right|^{2} d \mathbf{x}
$$


where equality holds only if $\left\{\sigma^{n}\right\}_{n=1}^{\infty}$ converges strongly in $L^{2}$ on the support of the test function $p_{j}$. In what follows we provide a formula for the limit on the left-hand side of (1.11). This formula is presented in Theorem 3.1 and is given in terms of derivatives of the G limit; see Sec. 2. Theorems 2.1, 2.2, and 2.3. It is pointed out that Theorem 2.1 provides an extension of the periodic localization principle [32] to the derivatives of $\mathrm{G}$ limits. Theorems 2.2 and 2.3 are in the spirit of [24] for the local representation of $G$ limits.

The tool used to establish Theorem 4.5 (the upper bound on the stress constrained $\mathrm{G}$ closure associated with (1.7)) is given by Theorem 3.2. In Theorem 3.2, a lower bound on the limit inferior of the $L^{\infty}$ norms of the stresses associated with $\mathrm{G}$ convergent sequences of elastic tensors is obtained. However, in order to precisely characterize the stress constrained $\mathrm{G}$ closure associated with (1.7), upper bounds on the limit superior of the $L^{\infty}$ norms of stresses associated with $\mathrm{G}$ convergent sequences of elastic tensors are required. The goal of future work will be to establish the necessary upper bounds when the limit superior of the $L^{\infty}$ norms of stresses is finite.

Earlier work [13] addresses the problem of optimal design in the context of thermal conductivity in the presence of a finite number of mean square constraints on the temperature gradient. As in the present case, the relaxed problem is given in terms of gradients of $\mathrm{G}$ limits. A related problem in which there has been recent development appears in the context of optimal design for multiphase conductors made from isotropic materials. The goal is to minimize the mean square deviation of the gradient from a prescribed target field. This problem is proposed in the work of [30] and requires new techniques. Here the extra difficulty is also due to a lack of continuity under weak convergence. For this problem it is the objective functional that is discontinuous with respect to weak convergence. In [30] a relaxation is obtained for a dense $G_{\delta}$ set of target fields in the space $W_{0}^{1,2}\left(\Omega, \mathbf{R}^{3}\right)$. This is accomplished through the introduction of the notion of strong $L^{2}$ closure. It is shown that minimizing sequences are exclusively associated with the well-known rank one laminates. Here the local layer orientation is parallel to the gradient field. As of this writing, this class of targets resists an explicit representation. However, numerical experiments using layered materials, see [14] and [31], suggest a conjecture that 0 lies in the $G_{\delta}$ set. For composites made of two isotropic phases, the work of [14] and [31] shows that minimizing sequences of configurations can be found within the class of rank one microstructures for any choice of target field. The more recent work [9] provides an explicit formula for the relaxation of the mean square deviation. This is used to rule out the appearance of minimizing sequences of layered configurations with more than one scale of oscillation and establishes that minimizing sequences of layered matcrials are exclusively given by rank one laminates. Another recent development is given in [23]. Here, for any choice of target, the notion of constrained quasiconvexity [22] is applied and is used to explicitly compute the constrained quasiconvex envelope of the mean square deviation [23]. This is also used to establish that minimizing sequences can be found within the class of rank 1 laminates as well as rule out the appearance of layered materials with more than one scale of oscillation.

In order to expedite the presentation, the following notation is used. The contractions of symmetric fourth order tensors $\mathbf{A}$ with second order tensors $\sigma$ are written $[A]_{i j k l} \sigma_{k l}=$ 
$\mathbf{A} \sigma$, contractions of two fourth order tensors $\mathbf{A}$ and $\mathbf{M}$ are written $[A]_{i j k l}[M]_{k l o p}=\mathbf{A M}$, contractions of two second order tensors $\sigma, \epsilon$ are written $\sigma_{i j} \epsilon_{i j}=\sigma: \epsilon, \sigma: \sigma=|\sigma|^{2}$, and contractions of second order tensors $\epsilon$ and vectors $\mathbf{x}$ are written $\epsilon_{i j} x_{j}=\epsilon \mathbf{x}$.

2. Local formulas for the derivatives of $\mathrm{G}$ limits and a local representation theorem. In this section, local formulas for the derivatives of $\mathrm{G}$ limits are provided as well as a local representation theorem for derivatives of $G$ limits. In order to define derivatives of $\mathrm{G}$ limits, we introduce the neighborhood $\mathcal{N}(\underline{\mathbf{A}})$ of the array $\underline{\mathbf{A}}$ of component elasticity tensors. Arrays in this neighborhood are denoted by $\underline{\mathbf{P}}=\left(\mathbf{P}_{1}, \mathbf{P}_{2}, \ldots, \mathbf{P}_{N}\right)$. The neighborhood is chosen such that all tensors $\mathbf{P}_{i}$ in the array satisfy the constraint $0<\lambda<\mathbf{P}_{i}<\Lambda$. The associated set of local elasticity tensors $\mathbf{C}(\underline{\mathbf{P}}, \mathbf{x})=\sum_{i=1}^{N} \chi_{i}(\mathbf{x}) \mathbf{P}_{i}$ for $\underline{\mathbf{P}}$ in $\mathcal{N}(\underline{\mathbf{A}})$ for which $\int_{\Omega} \chi_{i} d \mathbf{x} \leq \gamma_{i}$ is denoted by $\mathcal{C}(\underline{\mathbf{A}}, \gamma)$. The $\mathrm{G}$ convergence is given in the following definition [21].

Definition 2.1. The sequence of elasticity tensors $\left\{\mathbf{C}^{n}(\underline{\mathbf{A}}, \mathbf{x})\right\}_{n=1}^{\infty} \mathrm{G}$ converges to $\mathbf{C}^{E}(\underline{\mathbf{A}}, \mathbf{x})$ if and only if, for any open subset set $\omega$ of $\Omega$ and any $\mathbf{f}$ in $W^{-1,2}\left(\omega, \mathbf{R}^{3}\right)$, the solutions $\mathbf{u}^{n}$ in $W_{0}^{1,2}(\omega)$ of

$$
-\operatorname{div}\left(\mathbf{C}^{n}(\underline{\mathbf{A}}, \mathbf{x}) \epsilon\left(\mathbf{u}^{n}\right)\right)=\mathbf{f}
$$

converge to $\mathbf{u}$ weakly in $W_{0}^{1,2}(\omega)$ and

$$
\mathbf{C}^{n}(\underline{\mathbf{A}}, \mathbf{x}) \epsilon\left(\mathbf{u}^{n}\right) \rightarrow \mathbf{C}^{E}(\underline{\mathbf{A}}, \mathbf{x}) \epsilon(\mathbf{u})
$$

weakly in $L^{2}\left(\Omega, \mathbf{R}^{3 \times 3}\right)$, where $\mathbf{u}$ is the $W_{0}^{1,2}(\omega)$ solution of

$$
-\operatorname{div}\left(\mathbf{C}^{E}(\underline{\mathbf{A}}, \mathbf{x}) \epsilon(\mathbf{u})\right)=\mathbf{f} .
$$

The $\mathrm{G}$ limit is Lebesgue measurable with respect to the $\mathbf{x}$ variable [27]. Two fundamental compactness properties of $\mathrm{G}$ convergence are now stated.

PROPERTY 2.1. Given any sequence of coefficients $\left\{\mathbf{C}^{n}(\underline{\mathbf{A}}, \mathbf{x})\right\}_{n=1}^{\infty}$, there is a subsequence $\left\{\mathbf{C}^{n^{\prime}}(\underline{\mathbf{A}}, \mathbf{x})\right\}_{n^{\prime}=1}^{\infty}$ and an elasticity tensor $\mathbf{C}^{E}(\underline{\mathbf{A}}, \mathbf{x})$ such that $\left\{\mathbf{C}^{n^{\prime}}(\underline{\mathbf{A}}, \mathbf{x})\right\}_{n^{\prime}=1}^{\infty} \mathrm{G}$ converges to $\mathbf{C}^{E}(\underline{\mathbf{A}}, \mathbf{x})$.

This property is established for the case of symmetric coefficients in [27]. For the nonsymmetric case, see [21]; see also [28] for elliptic systems. This property extends to the elasticity tensor viewed as a function of $\underline{\mathbf{P}}$ on $\mathcal{N}(\underline{\mathbf{A}})$.

Property 2.2. Given a sequence $\left\{\mathbf{C}^{n}(\underline{\mathbf{P}}, \mathbf{x})\right\}_{n=1}^{\infty}$, then there exists a subsequence $\left\{\mathbf{C}^{n^{\prime}}(\underline{\mathbf{P}}, \mathbf{x})\right\}_{n^{\prime}=1}^{\infty}$ and an effective elasticity function $\mathbf{C}^{E}(\underline{\mathbf{P}}, \mathbf{x})$ such that $\left\{\mathbf{C}^{n^{\prime}}(\underline{\mathbf{P}}, \mathbf{x})\right\}_{n^{\prime}=1}^{\infty}$ $\mathrm{G}$ converges to $\mathbf{C}^{E}(\underline{\mathbf{P}}, \mathbf{x})$ for every $\underline{\mathbf{P}}$ in $\mathcal{N}(\underline{\mathbf{A}})$.

This property follows directly from [29].

In order to define local formulas for derivatives of $\mathrm{G}$ limits, we consider a sequence of configurations with elastic properties $\left\{\mathbf{C}^{n}(\underline{\mathbf{P}}, \mathbf{x})\right\}_{n=1}^{\infty}$ that $\mathrm{G}$ converge to the tensor $\mathbf{C}^{E}(\underline{\mathbf{P}}, \mathbf{x})$ for every $\underline{\mathbf{P}}$ in $\mathcal{N}(\underline{\mathbf{A}})$. Fix a cube $Q(\mathbf{x}, r)$ of side length $r$ centered at some point $\mathbf{x}$ in $\Omega$. For $r$ sufficiently small, $Q(\mathbf{x}, r)$ is contained within $\Omega$. Given a constant strain $\bar{\epsilon}$ applied to the cube, the local oscillatory response function is denoted by $\mathbf{w}_{\bar{\epsilon}}^{n, r}$, where $\mathbf{w}_{\bar{\epsilon}}^{n, r}$ is the $W_{\#}^{1,2}\left(Q(\mathbf{x}, r), \mathbf{R}^{3}\right)$ solution of

$$
-\operatorname{div}\left(\mathbf{C}^{n}(\underline{\mathbf{A}}, \mathbf{y})\left(\epsilon\left(\mathbf{w}_{\bar{\epsilon}}^{n, r}(\mathbf{y})\right)+\bar{\epsilon}\right)\right)=0 \text {, for } \mathbf{y} \text { in } Q(\mathbf{x}, r) \text {. }
$$


Here $W_{\#}^{1,2}\left(Q(\mathbf{x}, r), \mathbf{R}^{3}\right)$ is the subspace of functions in $W_{\text {loc }}^{1,2}\left(\mathbf{R}^{3}, \mathbf{R}^{3}\right)$ that are periodic with period cell $Q(\mathbf{x}, r)$ and zero mean when averaged over $Q(\mathbf{x}, r)$.

The local response functions are used to define directional derivatives of the $\mathrm{G}$ limit in the following theorem.

Theorem 2.1. The directional derivative of $\mathbf{C}^{E}(\underline{\mathbf{P}}, \mathbf{x})$ at $\underline{\mathbf{P}}=\underline{\mathbf{A}}$ with respect to the $i$ th component elasticity in the direction specified by the symmetric fourth order tensor $\mathbf{M}_{i}$ is given by

$$
\begin{aligned}
& \frac{\partial \mathbf{C}^{E}(\underline{\mathbf{A}}, \mathbf{x})}{\partial \mathbf{M}_{i}} \bar{\epsilon}: \bar{\epsilon} \\
& =\sum_{k=1}^{3} \sum_{l=1}^{3} \sum_{m=1}^{3} \sum_{n=1}^{3}\left[M_{i}\right]_{k l m n} \\
& \quad \times \lim _{r \rightarrow 0} \lim _{n \rightarrow \infty}(1 /|Q(\mathbf{x}, r)|) \int_{Q(\mathbf{x}, r)} \chi_{i}^{n}\left(\epsilon\left(\mathbf{w}_{\bar{\epsilon}}^{\varepsilon, r}\right)_{k \cdot l}+\bar{\epsilon}_{k l l}\right)\left(\epsilon\left(\mathbf{w}_{\bar{\epsilon}}^{\varepsilon, r}\right)_{m n}+\bar{\epsilon}_{m n}\right) d \mathbf{y},
\end{aligned}
$$

where $\bar{\epsilon}$ is any constant strain. The derivative is Lebesgue measurable with respect to $\mathbf{x}$.

It follows that the definition of the $i$ th phase gradient denoted by $\left(\nabla_{k l m n}^{i} \mathbf{C}^{E}(\underline{\mathbf{A}}, \mathbf{x})\right) \bar{\epsilon}: \bar{\epsilon}$ is given by the local formula

$$
\begin{aligned}
& \left(\nabla_{k l m n}^{i} \mathbf{C}^{E}(\underline{\mathbf{A}}, \mathbf{x})\right) \bar{\epsilon}: \bar{\epsilon} \\
& =\lim _{r \rightarrow 0} \lim _{n \rightarrow \infty}(1 /|Q(\mathbf{x}, r)|) \int_{Q(\mathbf{x}, r)} \chi_{i}^{\varepsilon}\left(\epsilon\left(\mathbf{w}_{\bar{\epsilon}}^{\varepsilon, r}\right)_{k l}+\bar{\epsilon}_{k l}\right)\left(\epsilon\left(\mathbf{w}_{\bar{\epsilon}}^{\varepsilon, r}\right)_{m n}+\bar{\epsilon}_{m n}\right) d \mathbf{y}
\end{aligned}
$$

and

$$
\left(\nabla_{k l k l}^{i} \mathbf{C}^{E}(\underline{\mathbf{A}}, \mathbf{x})\right) \bar{\epsilon}: \bar{\epsilon}=\lim _{r \rightarrow 0} \lim _{n \rightarrow \infty}(1 /|Q(\mathbf{x}, r)|) \int_{Q(\mathbf{x}, r)} \chi_{i}^{\varepsilon}\left(\epsilon\left(\mathbf{w}_{\bar{\epsilon}}^{\varepsilon, r}\right)_{k l}+\bar{\epsilon}_{k l}\right)\left(\epsilon\left(\mathbf{w}_{\bar{\epsilon}}^{\varepsilon, r}\right)_{k l}+\bar{\epsilon}_{k l}\right) d \mathbf{y},
$$

where repeated indices indicate summation. The formula for the $\mathrm{G}$ limit is

$$
\mathbf{C}^{E}(\underline{\mathbf{A}}, \mathbf{x}) \bar{\epsilon}: \bar{\epsilon}=\lim _{r \rightarrow 0} \lim _{n \rightarrow x}(1 /|Q(\mathbf{x}, r)|) \int_{Q(\mathbf{x}, r)} \mathbf{C}^{n}(\underline{\mathbf{A}}, \mathbf{y})\left(\epsilon\left(\mathbf{w}_{\bar{\epsilon}}^{n, r}\right)+\bar{\epsilon}\right):\left(\epsilon\left(\mathbf{w}_{\bar{\epsilon}}^{n, r}\right)+\bar{\epsilon}\right) d \mathbf{y} .
$$

The local formula (2.8) for the $G$ limit is known; see [27] and [32]. In fact, formula (2.8) holds true when the response functions are chosen from any space $V$ such that $W_{0}^{1,2}\left(Q(\mathbf{x}, r), \mathbf{R}^{3}\right) \subseteq V \subseteq \widetilde{W}^{1.2}\left(Q(\mathbf{x}, r), \mathbf{R}^{3}\right)$, where

$$
\widetilde{W}^{1,2}\left(Q(\mathbf{x}, r), \mathbf{R}^{3}\right)=\left\{\mathbf{v} \text { in } W^{1,2}\left(Q(x, r), \mathbf{R}^{3}\right): \int_{Q(\mathbf{x}, r)} \epsilon(\mathbf{v}(\mathbf{y})) d \mathbf{y}=0\right\} .
$$

This follows directly from Remarks 13, 17, and 18 of [27]. The formulas (2.5) and (2.6) for the derivatives of the effective elastic tensor are semi-explicit in that they are given in terms of sequences of solutions to local problems. These formulas hold for general oscillations and are obtained without any hypotheses on the sequence of configurations. They provide the extension of the concept of periodic localization put forth in [32] to the derivatives of $\mathrm{G}$ limits. 
We present a local representation of the set of all $G$ limits and their derivatives. Consider a $\mathrm{G}$ limit $\mathbf{C}^{E}(\underline{\mathbf{A}}, \mathbf{x})$ together with its phase gradients

$$
\vec{\nabla}^{i} \mathbf{C}^{E}(\underline{\mathbf{A}}, \mathbf{x}) \stackrel{\text { def }}{=} \nabla_{k l m n}^{i} \mathbf{C}^{E}(\underline{\mathbf{A}}, \mathbf{x})_{o p q r}
$$

and denote them by the array $\left(\mathbf{C}^{E}(\underline{\mathbf{A}}, \mathbf{x}), \partial \mathbf{C}^{E}(\underline{\mathbf{A}}, \mathbf{x})\right)$, defined by

$$
\left(\mathbf{C}^{E}(\underline{\mathbf{A}}, \mathbf{x}), \partial \mathbf{C}^{E}(\underline{\mathbf{A}}, \mathbf{x})\right) \stackrel{\text { def }}{=}\left(\mathbf{C}^{E}(\underline{\mathbf{A}}, \mathbf{x}), \vec{\nabla}^{1} \mathbf{C}^{E}(\underline{\mathbf{A}}, \mathbf{x}), \ldots, \vec{\nabla}^{N} \mathbf{C}^{E}(\underline{\mathbf{A}}, \mathbf{x})\right) .
$$

We provide a characterization of the array $\left(\mathbf{C}^{E}(\underline{\mathbf{A}}, \mathbf{x}), \partial \mathbf{C}^{E}(\underline{\mathbf{A}}, \mathbf{x})\right)$ for almost all $\mathbf{x}$ in $\Omega$. To start, we consider any partition of the unit cube into $N$ Lebesgue measurable subsets. The indicator functions of these subsets are denoted by $\chi_{i}, i=1, \ldots, N$. Given a vector $\underline{\theta}=\left(\theta_{1}, \theta_{2}, \ldots, \theta_{N}\right)$ such that $0 \leq \theta_{i} \leq 1$ and $\sum_{i=1}^{N} \theta_{i}=1$, the set $S_{\underline{\theta}}(\underline{\mathbf{A}})$ is the set of all arrays $\left(\mathbf{C}^{H}(\underline{\mathbf{A}}), \partial \mathbf{C}^{H}(\underline{\mathbf{A}})\right)$ such that there exists a $\mathbf{C}_{0}(\underline{\mathbf{P}}, \mathbf{y})=\sum_{i=1}^{N} \chi_{i}(\mathbf{y}) \mathbf{P}_{i}$ with $\underline{\mathbf{P}}$ in $\mathcal{N}(\underline{\mathbf{A}}), \int_{Q(0.1)} \chi_{i} d \mathbf{y}=\theta_{i}$ and

$$
\begin{aligned}
\mathbf{C}^{H}(\underline{\mathbf{A}}) \bar{\epsilon}: \bar{\epsilon} & =\int_{Q(0,1)} \mathbf{C}_{0}(\underline{\mathbf{A}}, \mathbf{y})(\epsilon(\mathbf{w})+\bar{\epsilon}):(\epsilon(\mathbf{w})+\bar{\epsilon}) d \mathbf{y} \\
\vec{\nabla}^{i} \mathbf{C}^{H}(\underline{\mathbf{A}}) \bar{\epsilon}: \bar{\epsilon} & =\int_{Q(0,1)} \chi_{i}(\mathbf{y})(\epsilon(\mathbf{w})+\bar{\epsilon}) \otimes(\epsilon(\mathbf{w})+\bar{\epsilon}) d \mathbf{y}, i=1, \ldots, N,
\end{aligned}
$$

where $\mathbf{w}$ is the $W_{\#}^{1,2}\left(Q(0,1), \mathbf{R}^{3}\right)$ solution of

$$
-\operatorname{div}\left(\mathbf{C}_{0}(\underline{\mathbf{A}}, \mathbf{y})(\epsilon(\mathbf{w}(\mathbf{y}))+\bar{\epsilon})\right)=0
$$

for any constant strain $\bar{\epsilon}$. For $\underline{\mathbf{P}}$ in $\mathcal{N}(\underline{\mathbf{P}})$, the function $\mathbf{C}^{H}(\underline{\mathbf{P}})$ is given by

$$
\mathbf{C}^{H}(\underline{\mathbf{P}}) \bar{\epsilon}: \bar{\epsilon}=\int_{Q(0,1)} \mathbf{C}_{0}(\underline{\mathbf{P}}, \mathbf{y})(\epsilon(\mathbf{w})+\bar{\epsilon}):(\epsilon(\mathbf{w})+\bar{\epsilon}) d \mathbf{y}
$$

where

$$
-\operatorname{div}\left(\mathbf{C}_{0}(\underline{\mathbf{P}}, \mathbf{y})(\epsilon(\mathbf{w}(\mathbf{y}))+\bar{\epsilon})\right)=0 .
$$

Direct calculation (as in the proof of Theorem 2.1) shows that the derivatives of $\mathbf{C}^{H}(\underline{\mathbf{P}})$ at $\underline{\mathbf{P}}=\underline{\mathbf{A}}$ are given by $(2.12)$.

Theorem 2.2. For almost every $\mathbf{x}$ in $\Omega$, the array $\left(\mathbf{C}^{E}(\underline{\mathbf{A}}, \mathbf{x}), \partial \mathbf{C}^{E}(\underline{\mathbf{A}}, \mathbf{x})\right)$ lies in the closure of $S_{\underline{\theta}}(\underline{\mathbf{A}})$.

The converse of Theorem 2.2 is given by

THEOREM 2.3. Given the measurable functions $\theta_{i}(\mathbf{x}), i=1, \ldots, N$, such that $\sum_{i} \theta_{i}(\mathbf{x})=$ $1, \int_{\Omega} \theta_{i}(\mathbf{x}) d \mathbf{x} \leq \gamma_{i}$, suppose that for every $\mathbf{x}$ in $\Omega$ that the measurable tensor $\overline{\mathbf{C}}(\underline{\mathbf{P}}, \mathbf{x})$ is a limit of tensors of the form (2.14) and that the array of measurable tensors $(\overline{\mathbf{C}}(\underline{\mathbf{A}}, \mathbf{x})$, $\partial \overline{\mathbf{C}}(\underline{\mathbf{A}}, \mathbf{x}))$ takes values in the closure of $S_{\underline{\theta}(\mathbf{x})}(\underline{\mathbf{A}})$, then there exists a sequence $\left\{\mathbf{C}^{n}(\underline{\mathbf{P}}, \mathbf{x})\right\}_{n=1}^{\infty} \subset \mathcal{C}(\underline{\mathbf{A}}, \underline{\gamma})$ such that $\left\{\mathbf{C}^{n}(\underline{\mathbf{P}}, \mathbf{x})\right\}_{n=1}^{\infty} \mathrm{G}$ converges to $\overline{\mathbf{C}}(\underline{\mathbf{P}}, \mathbf{x})$ for all $\underline{\mathbf{P}}$ in $\mathcal{N}(\underline{\mathbf{A}})$.

The proofs of Theorems 2.1, 2.2, and 2.3 are given in the following subsections. 
2.1. Proof of Theorem 2.1. In this subsection the formula for derivative of the $\mathrm{G}$ limit is established. Consider a sequence $\left\{\mathbf{C}^{n}(\underline{\mathbf{P}}, \mathbf{x})\right\}_{n=1}^{\infty}$ that $\mathrm{G}$ converges to $\mathbf{C}^{E}(\underline{\mathbf{P}}, \mathbf{x})$ for every $\underline{\mathbf{P}}$ in $\mathcal{N}(\underline{\mathbf{A}})$. Given a constant strain $\bar{\epsilon}$, the local oscillatory response $\mathbf{w}_{\bar{\epsilon}}^{n, r}$ is the $W_{\#}^{1,2}\left(Q(\mathbf{x}, r) ; \mathbf{R}^{3}\right)$ solution of (2.4). The differential equation (2.4) is written in the weak form given by

$$
\int_{Q(\mathbf{x}, r)} \mathbf{C}^{n}(\underline{\mathbf{A}}, \mathbf{x})\left(\epsilon\left(\mathbf{w}_{\bar{\epsilon}}^{n, r}\right)+\bar{\epsilon}\right): \epsilon(\mathbf{v}) d \mathbf{y}=0,
$$

for all $\mathbf{v}$ in $W_{\#}^{1,2}\left(Q(\mathbf{x}, r) ; \mathbf{R}^{3}\right)$. The volume of $Q(\mathbf{x}, r)$ is denoted by $|Q(\mathbf{x}, r)|$ and an application of Cauchy's inequality in (2.16) gives

$$
\int_{Q(\mathbf{x}, r)}\left|\epsilon\left(\mathbf{w}_{\bar{\epsilon}}^{n, r}\right)\right|^{2} d \mathbf{y} \leq\left(\Lambda^{2} / \lambda^{2}\right)|Q(\mathbf{x}, r)||\bar{\epsilon}|^{2},
$$

and

$$
\int_{Q(\mathbf{x}, r)}\left|\epsilon\left(\mathbf{w}_{\bar{\epsilon}}^{n}\right)+\bar{\epsilon}\right|^{2} d \mathbf{y} \leq\left(\Lambda^{2} / \lambda^{2}+1\right)|Q(\mathbf{x}, r)||\bar{\epsilon}|^{2} .
$$

Choose a number $\delta \beta$ and $\underline{\mathbf{M}}$ such that $\underline{\mathbf{A}}+\delta \beta \underline{\mathbf{M}}$ are in $\mathcal{N}(\underline{\mathbf{A}})$ and consider the sequence $\left\{\mathbf{C}^{n}(\underline{\mathbf{A}}+\delta \beta \underline{\mathbf{M}}, x)\right\}_{n=1}^{\infty}$. Here $\underline{\mathbf{M}}$ is an array of elastic tensors that is identically zero except for the $i$ th component elastic tensor $\mathbf{M}_{i}$. Here $\mathbf{M}_{i}$ is of norm one where the tensor norm is given by $\left|\mathbf{M}_{i}\right|=\sqrt{\sum_{k, l, m, n}\left[M_{i}\right]_{k l m n}^{2}}$. The sequence of coefficients $\left\{\mathbf{C}^{n}(\underline{\mathbf{A}}+\delta \beta \underline{\mathbf{M}}, \mathbf{x})\right\}_{n=1}^{\infty}$ differs from $\left\{\mathbf{C}^{n}(\underline{\mathbf{A}}, \mathbf{x})\right\}_{n=1}^{\infty}$ by the increment $\delta \beta \mathbf{M}_{i} \chi_{i}^{n}$. The $\mathrm{G}$ limit for the sequence $\left\{\mathbf{C}^{n}(\underline{\mathbf{A}}+\delta \beta \underline{\mathbf{M}}, \mathbf{x})\right\}_{n=1}^{\infty}$ is written as $\mathbf{C}^{E}(\underline{\mathbf{A}}+\delta \beta \underline{\mathbf{M}}, \mathbf{x})$. We set $\delta \mathbf{C}=\mathbf{C}^{E}(\underline{\mathbf{A}}+\delta \beta \underline{\mathbf{M}}, \mathbf{x})-$ $C^{E}(\underline{\mathbf{A}}, \mathbf{x})$ and use $(2.8)$ to compute $\delta \mathbf{C}$ with respect to the increment $\delta \beta$. The oscillatory responses associated with the sequence $\left\{\mathbf{C}^{n}(\underline{\mathbf{A}}+\delta \beta \underline{\mathbf{M}}, \mathbf{x})\right\}_{n=1}^{\infty}$ are denoted by $\widehat{\mathbf{w}}_{\bar{\epsilon}}^{n . r}$, where $\widehat{\mathbf{w}}_{\bar{\epsilon}}^{n, r}$ are $W_{\#}^{1,2}\left(Q(\mathbf{x}, r) ; \mathbf{R}^{3}\right)$ solutions of

$$
\int_{Q(\mathbf{x}, r)} \mathbf{C}^{n}(\underline{\mathbf{A}}+\delta \beta \underline{\mathbf{M}}, \mathbf{y})\left(\epsilon\left(\hat{\mathbf{w}}_{\bar{\epsilon}}^{n, r}\right)+\bar{\epsilon}\right): \epsilon(\mathbf{v}) d \mathbf{y}=0,
$$

for all $\mathbf{v}$ in $W_{\#}^{1,2}\left(Q(\mathbf{x}, r) ; \mathbf{R}^{3}\right)$.

It follows from (2.8) that

$$
\begin{aligned}
\delta \mathbf{C} \bar{\epsilon}: \bar{\epsilon}= & \lim _{r \rightarrow 0} \lim _{n \rightarrow \infty}(1 /|Q(\mathbf{x}, r)|) \int_{Q(\mathbf{x}, r)} \mathbf{C}^{n}(\underline{\mathbf{A}}+\delta \beta \underline{\mathbf{M}}, \mathbf{y})\left(\epsilon\left(\hat{\mathbf{w}}_{\bar{\epsilon}}^{n, r}\right)+\bar{\epsilon}\right):\left(\epsilon\left(\hat{\mathbf{w}}_{\bar{\epsilon}}^{n, r}\right)+\bar{\epsilon}\right) d \mathbf{y} \\
& \left.-\lim _{r \rightarrow 0} \lim _{n \rightarrow \infty}(1 /|Q(\mathbf{x}, r)|) \int_{Q(\mathbf{x}, r)} \mathbf{C}^{n}(\underline{\mathbf{A}}, \mathbf{y})\left(\epsilon\left(\mathbf{w}_{\bar{\epsilon}}^{n, r}\right)+\bar{\epsilon}\right):\left(\mathbf{w}_{\bar{\epsilon}}^{n, r}\right)+\bar{\epsilon}\right) d \mathbf{y} . \quad(2.20)
\end{aligned}
$$

Writing $\mathbf{C}^{n}(\underline{\mathbf{A}}+\delta \beta \underline{\mathbf{M}}, \mathbf{y})=\mathbf{C}^{n}(\underline{\mathbf{A}}, \mathbf{y})+\delta \beta \mathbf{M}_{i} \chi_{i}^{n}$ and $\hat{\mathbf{w}}_{\bar{\epsilon}}^{n, r}=\mathbf{w}_{\bar{\epsilon}}^{n, r}+\delta \mathbf{w}^{n, r}$, where $\delta \mathbf{w}^{n, r}=\hat{\mathbf{w}}_{\bar{\epsilon}}^{n, r}-\mathbf{w}_{\bar{\epsilon}}^{n, r}$, one has, for every $\mathbf{v}$ in $W_{\#}^{1,2}\left(Q(\mathbf{x}, r) ; \mathbf{R}^{3}\right)$, the equation

$$
0=\int_{Q(\mathbf{x}, r)} \delta \beta \mathbf{M}_{i} \chi_{i}^{n}\left(\epsilon\left(\mathbf{w}_{\bar{\epsilon}}^{n, r}\right)+\bar{\epsilon}\right): \epsilon(\mathbf{v}) d \mathbf{y}+\int_{Q(\mathbf{x}, r)} \mathbf{C}^{n}(\underline{\mathbf{A}}+\delta \beta \underline{\mathbf{M}}, \mathbf{y}) \epsilon\left(\delta \mathbf{w}^{n, r}\right): \epsilon(\mathbf{v}) d \mathbf{y}
$$


Setting $\mathbf{v}=\delta \mathbf{w}^{n, r}$ in (2.21) and substitution into (2.20) gives

$\delta \mathbf{C} \bar{\epsilon}: \bar{\epsilon}$

$$
\begin{aligned}
= & \delta \beta \lim _{r \rightarrow 0} \lim _{n \rightarrow \infty} \\
\times & (1 /|Q(\mathbf{x}, r)|) \int_{Q(\mathbf{x}, r)}\left(\mathbf{M}_{i} \chi_{i}^{n}\left(\epsilon\left(\mathbf{w}_{\bar{\epsilon}}^{n, r}\right)+\bar{\epsilon}\right):\left(\epsilon\left(\mathbf{w}_{\bar{\epsilon}}^{n, r}\right)+\bar{\epsilon}\right)\right. \\
& \left.+\mathbf{M}_{i} \chi_{i}^{n}\left(\epsilon\left(\mathbf{w}_{\bar{\epsilon}}^{n, r}\right)+\bar{\epsilon}\right): \epsilon\left(\delta \mathbf{w}^{n, r}\right)\right) d \mathbf{y} .
\end{aligned}
$$

Setting $\mathbf{v}=\delta \mathbf{w}^{n, r}$ in (2.21) and application of Cauchy's inequality gives

$$
\int_{Q(\mathbf{x}, r)}\left|\epsilon\left(\delta \mathbf{w}^{n, r}\right)\right|^{2} d \mathbf{y} \leq \delta \beta^{2} \lambda^{-2}|Q(\mathbf{x}, r)||\bar{\epsilon}|^{2}\left(1+(\Lambda / \lambda)^{2}\right) .
$$

Estimates (2.18) and (2.23) show that

$$
|(1 / \mid Q(\mathbf{x}, r))| \int_{Q(\mathbf{x}, r)} \mathbf{M}_{i} \chi_{i}^{n}\left(\epsilon\left(\mathbf{w}_{\bar{\epsilon}}^{n, r}\right)+\bar{\epsilon}\right):\left.\epsilon\left(\delta \mathbf{w}^{n, r}\right) d \mathbf{y}|\leq \delta \beta| \bar{\epsilon}\right|^{2}\left(1+(\Lambda / \lambda)^{2}\right) .
$$

From this one deduces that

$$
\delta \mathbf{C} \bar{\epsilon}: \bar{\epsilon}=\delta \beta \lim _{r \rightarrow 0} \lim _{n \rightarrow \infty}(1 /|Q(\mathbf{x}, r)|) \int_{Q(\mathbf{x}, r)} \mathbf{M}_{i} \chi_{i}^{n}\left(\epsilon\left(\mathbf{w}_{\bar{\epsilon}}^{n, r}\right)+\bar{\epsilon}\right):\left(\epsilon\left(\mathbf{w}_{\bar{\epsilon}}^{n, r}\right)+\bar{\epsilon}\right) d \mathbf{y}+o(\delta \beta)
$$

and (2.5) follows. The measurability of $\partial \mathbf{C}^{E}(\underline{\mathbf{A}}, \mathbf{x}) / \partial \underline{\mathbf{M}}_{i}$ is assured as it is a pointwise limit of measurable functions and Theorem 2.1 follows.

2.2. Proof of Theorem 2.2. Consider a sequence of configurations with elastic properties $\left\{\mathbf{C}^{n}(\underline{\mathbf{P}}, \mathbf{x})\right\}_{n=1}^{\infty}$ in $\mathcal{C}(\underline{\mathbf{A}}, \underline{\gamma})$ that $\mathrm{G}$ converge to the effective tensor $\mathbf{C}^{E}(\underline{\mathbf{P}}, \mathbf{x})$ for every $\underline{\mathbf{P}}$ in $\mathcal{N}(\underline{\mathbf{A}})$ and with characteristic functions $\left\{\chi_{i}^{n}\right\}_{n=1}^{\infty}$ converging to $\theta_{i}$ in $L^{\infty}$ weak *. Then for any cube $Q(\mathbf{x}, r)$ contained in $\Omega$, one has

$$
\lim _{n \rightarrow \infty}(1 /|Q(\mathbf{x}, r)|) \int_{Q(\mathbf{x}, r)} \chi_{i}^{n} d \mathbf{y}=(1 /|Q(\mathbf{x}, r)|) \int_{Q(\mathbf{x}, r)} \theta_{i} d \mathbf{y} .
$$

At the Lebesgue points of $\theta_{i}$, one sees that

$$
\lim _{r \rightarrow 0} \lim _{n \rightarrow \infty}(1 /|Q(\mathbf{x}, r)|) \int_{Q(\mathbf{x}, r)} \chi_{i}^{n} d \mathbf{y}=\theta_{i}(\mathbf{x}) .
$$

Starting with the formulas (2.6), (2.8), and (2.27), we choose a diagonal sequence indexed by $j$ such that $r_{j} \rightarrow 0$ and $n_{j} \rightarrow \infty$ as $j \rightarrow \infty$, for which

$$
\begin{gathered}
\left(\vec{\nabla}^{i} \mathbf{C}^{E}(\underline{\mathbf{A}}, \mathbf{x})\right) \bar{\epsilon}: \bar{\epsilon}=\lim _{j \rightarrow \infty}\left(1 /\left|Q\left(\mathbf{x}, r_{j}\right)\right|\right) \int_{Q\left(\mathbf{x}, r_{j}\right)} \chi_{i}^{n_{j}}\left(\epsilon\left(\mathbf{w}_{\bar{\epsilon}}^{j}\right)+\bar{\epsilon}\right) \otimes\left(\epsilon\left(\mathbf{w}_{\bar{\epsilon}}^{j}\right)+\bar{\epsilon}\right) d \mathbf{y}, \\
\mathbf{C}^{E}(\underline{\mathbf{A}}, \mathbf{x}) \bar{\epsilon}: \bar{\epsilon}=\lim _{j \rightarrow \infty}\left(1 /\left|Q\left(\mathbf{x}, r_{j}\right)\right|\right) \int_{Q\left(\mathbf{x}, r_{j}\right)} \mathbf{C}^{n_{j}}(\underline{\mathbf{A}}, \mathbf{y})\left(\epsilon\left(\mathbf{w}_{\bar{\epsilon}}^{j}\right)+\bar{\epsilon}\right):\left(\epsilon\left(\mathbf{w}_{\bar{\epsilon}}^{j}\right)+\bar{\epsilon}\right) d \mathbf{y}
\end{gathered}
$$

and

$$
\theta_{i}(\mathbf{x})=\lim _{j \rightarrow \infty}\left(1 /\left|Q\left(\mathbf{x}, r_{j}\right)\right|\right) \int_{Q\left(\mathbf{x}, r_{j}\right)} \chi_{i}^{n_{j}} d \mathbf{y} .
$$

Here the local oscillatory response function is denoted by $\mathbf{w}_{\bar{\epsilon}}^{j}$, where $\mathbf{w}_{\bar{\epsilon}}^{j}$ is the $W_{\#}^{1,2}\left(Q\left(\mathbf{x}, r_{j}\right), \mathbf{R}^{3}\right)$ solution of

$$
-\operatorname{div}\left(\mathbf{C}^{n_{j}}(\underline{\mathbf{A}}, \mathbf{y})\left(\epsilon\left(\mathbf{w}_{\bar{\epsilon}}^{j}(\mathbf{y})\right)+\bar{\epsilon}\right)\right)=0, \text { for } \mathbf{y} \text { in } Q\left(\mathbf{x}, r_{j}\right) .
$$


To prove Theorem 2.2 we change coordinates so that $\mathbf{x}=0$ and show that $\left(\mathbf{C}^{E}(\underline{\mathbf{A}}, 0), \partial \mathbf{C}^{E}(\underline{\mathbf{A}}, 0)\right)$ lies in the closure of $S_{\underline{\theta}}(\underline{\mathbf{A}})$. Using the rescaled variable $\mathbf{z}=\mathbf{y} / r_{j}$ and writing $\mathbf{w}_{\bar{\epsilon}}^{j}(\mathbf{y})=r_{j} \phi_{\bar{\epsilon}}^{j}\left(\mathbf{y} / r_{j}\right)$, where $\phi_{\bar{\epsilon}}^{j}(\mathbf{z})$ is the $W_{\#}^{1.2}\left(Q(0,1) ; \mathbf{R}^{3}\right)$ solution of

$$
-\operatorname{div}\left(\mathbf{C}^{n_{j}}\left(\underline{\mathbf{A}}, r_{j} \mathbf{z}\right)\left(\epsilon\left(\phi_{\bar{\epsilon}}^{j}(\mathbf{z})\right)+\bar{\epsilon}\right)\right)=0, \text { for } \mathbf{z} \text { in } Q(0,1),
$$

it is seen that

$$
\begin{gathered}
\left(\vec{\nabla}^{i} \mathbf{C}^{E}(\underline{\mathbf{A}}, 0)\right) \bar{\epsilon}: \bar{\epsilon}=\lim _{j \rightarrow \infty} \int_{Q(0,1)} \chi_{i}^{n_{j}}\left(r_{j} \mathbf{z}\right)\left(\epsilon\left(\phi_{\bar{\epsilon}}^{j}\right)+\bar{\epsilon}\right) \otimes\left(\epsilon\left(\phi_{\bar{\epsilon}}^{j}\right)+\bar{\epsilon}\right) d \mathbf{z}, \\
\mathbf{C}^{E}(\underline{\mathbf{A}}, 0) \bar{\epsilon}: \bar{\epsilon}=\lim _{j \rightarrow \infty} \int_{Q(0,1)} \mathbf{C}^{n_{j}}\left(\underline{\mathbf{A}}, r_{j} \mathbf{z}\right)\left(\epsilon\left(\phi_{\bar{\epsilon}}^{j}\right)+\bar{\epsilon}\right):\left(\epsilon\left(\phi_{\bar{\epsilon}}^{j}\right)+\bar{\epsilon}\right) d \mathbf{z}
\end{gathered}
$$

and

$$
\theta_{i}(0)=\lim _{j \rightarrow \infty} \int_{Q(0,1)} \chi_{i}^{n_{j}}\left(r_{j} \mathbf{z}\right) d \mathbf{z}
$$

To conclude the proof we show that, for sufficiently large indices $j$, we can slightly modify $\chi_{i}^{n_{j}}$ so that its modification $\tilde{\chi}_{i}^{n_{j}}$ satisfies

$$
\theta_{i}(0)=\int_{Q(0,1)} \tilde{\chi}_{i}^{n_{j}}\left(r_{j} \mathbf{z}\right) d \mathbf{z}
$$

for every $j$ greater than some $J$ and

$$
\begin{aligned}
& \left(\vec{\nabla}^{i} \mathbf{C}^{E}(\underline{\mathbf{A}}, 0)\right) \bar{\epsilon}: \bar{\epsilon}=\lim _{j \rightarrow \infty} \int_{Q(0.1)} \tilde{\chi}_{i}^{n_{j}}\left(r_{j} \mathbf{z}\right)\left(\epsilon\left(\tilde{\phi}_{\bar{\epsilon}}^{j}\right)+\bar{\epsilon}\right) \otimes\left(\epsilon\left(\tilde{\phi}_{\bar{\epsilon}}^{j}\right)+\bar{\epsilon}\right) d \mathbf{z}, \\
& \mathbf{C}^{E}(\underline{\mathbf{A}}, 0) \bar{\epsilon}: \bar{\epsilon}=\lim _{j \rightarrow \infty} \int_{Q(0,1)} \tilde{\mathbf{C}}^{n_{j}}\left(\underline{\mathbf{A}}, r_{j} \mathbf{z}\right)\left(\epsilon\left(\tilde{\phi}_{\bar{\epsilon}}^{j}\right)+\bar{\epsilon}\right):\left(\epsilon\left(\tilde{\phi}_{\bar{\epsilon}}^{j}\right)+\bar{\epsilon}\right) d \mathbf{z},
\end{aligned}
$$

where

$$
-\operatorname{div}\left(\widetilde{\mathbf{C}}^{n_{j}}\left(\underline{\mathbf{A}}, r_{j} \mathbf{z}\right)\left(\epsilon\left(\tilde{\phi}_{\bar{\epsilon}}^{j}(\mathbf{z})\right)+\bar{\epsilon}\right)\right)=0, \text { for } \mathbf{z} \text { in } Q(0,1),
$$

$\tilde{\phi}_{\bar{\epsilon}}^{j}$ is in $W_{\#}^{1.2}\left(Q(\mathbf{x}, r) ; \mathbf{R}^{3}\right)$, and $\widetilde{\mathbf{C}}^{n_{j}}\left(\underline{\mathbf{P}}, r_{j} \mathbf{z}\right)=\sum_{i=1}^{N} \tilde{\chi}_{i}^{n_{j}}\left(\mathbf{r}_{j} \mathbf{z}\right) \mathbf{P}_{i}$.

Since $\theta_{i}^{r}=\int_{Q(0,1)} \chi_{i}^{n_{j}}\left(r_{j} \mathbf{z}\right) d \mathbf{z} \rightarrow \theta_{i}(0)$, it is evident that we can introduce modifications $\tilde{\chi}_{i}^{n_{j}}\left(r_{j} \mathbf{z}\right)$ for which

$$
\int_{Q(0,1)}\left|\tilde{\chi}_{i}^{n_{j}}\left(r_{j} \mathbf{z}\right)-\chi_{i}^{n_{j}}\left(r_{j} \mathbf{z}\right)\right| d \mathbf{z} \rightarrow 0 \text { as } j \rightarrow \infty
$$

and for some $J$ that

$$
\int_{Q(0,1)} \tilde{\chi}_{i}^{n_{j}}\left(r_{j} \mathbf{z}\right) d \mathbf{z}=\theta_{i}(0)
$$

for every $j>J$. An application of the higher integrability result of Meyers and Elcrat [18] together with Hölder's inequality shows that the convergence given by $(2.40)$ is sufficient to guarantee the strong $L^{2}\left(Q(0,1) ; \mathbf{R}^{3 \times 3}\right)$ convergence of the difference $\epsilon\left(\tilde{\phi}_{\bar{\epsilon}}^{j}\right)-\epsilon\left(\phi_{\bar{\epsilon}}^{j}\right)$ and Theorem 2.2 follows. 
2.3. Proof of Theorem 2.3. Given a set of functions $\theta_{i}, i=1, \ldots, N$, and $\overline{\mathbf{C}}(\underline{\mathbf{P}}, \mathbf{x})$ satisfying the hypothesis of Theorem 2.3 , we suppose first that the array $(\overline{\mathbf{C}}(\underline{\mathbf{A}}, \mathbf{x}), \partial \overline{\mathbf{C}}(\underline{\mathbf{A}}, \mathbf{x}))$ takes values in $S_{\underline{\theta}(\mathbf{x})}(\underline{\mathbf{A}})$ and establish the theorem for this case.

Since $(\overline{\mathbf{C}}(\underline{\mathbf{A}}, \mathbf{x}), \partial \overline{\mathbf{C}}(\underline{\mathbf{A}}, \mathbf{x})) \in S_{\underline{\theta}(\mathbf{x})}(\underline{\mathbf{A}})$, it follows that for each $\mathbf{x}$ in $\Omega$ the associated function $\overline{\mathbf{C}}(\underline{\mathbf{P}}, \mathbf{x})$ is defined for all $\underline{\mathbf{P}}$ in $\mathcal{N}(\underline{\mathbf{A}})$ in terms of a simple function defined on $Q(0,1)$ indexed by $\mathbf{x}$ and denoted by $\overline{\mathbf{C}}_{0}(\underline{\mathbf{P}}, \mathbf{y}, \mathbf{x})=\sum_{i=1}^{N} \chi_{i}(\mathbf{y}, \mathbf{x}) \mathbf{P}_{i}$, where

$$
\overline{\mathbf{C}}(\underline{\mathbf{P}}, \mathbf{x}) \bar{\epsilon}: \bar{\epsilon}=\int_{Q(0,1)} \overline{\mathbf{C}}_{0}(\underline{\mathbf{P}}, \mathbf{y}, \mathbf{x})(\epsilon(\mathbf{w})+\bar{\epsilon}):(\epsilon(\mathbf{w})+\bar{\epsilon}) d \mathbf{y} .
$$

Here $\mathbf{w}$ is the $W_{\#}^{1,2}\left(Q(0,1), \mathbf{R}^{3}\right)$ solution of

$$
-\operatorname{div}\left(\overline{\mathbf{C}}_{0}(\underline{\mathbf{A}}, \mathbf{y}, \mathbf{x})(\epsilon(\mathbf{w}(\mathbf{y}))+\bar{\epsilon})\right)=0
$$

for any constant strain $\bar{\epsilon}$. In order to establish the theorem for this case, it suffices to show that there exists a sequence in $\left\{\mathbf{C}^{n}(\underline{\mathbf{P}}, \mathbf{x})\right\}_{n=1}^{\infty} \subset \mathcal{C}(\underline{\mathbf{A}}, \gamma)$ that $\mathrm{G}$ converges to $\overline{\mathbf{C}}(\underline{\mathbf{P}}, \mathbf{x})$ for all $\underline{\mathbf{P}}$ in $\mathcal{N}(\underline{\mathbf{A}})$. Repeated application of Lusin's theorem together with the differentiability of $\overline{\mathbf{C}}(\underline{\mathbf{P}}, \mathbf{x})$ with respect to $\underline{\mathbf{P}}$ in $\mathcal{N}(\underline{\mathbf{A}})$ delivers the approximation to $\overline{\mathbf{C}}(\underline{\mathbf{P}}, \mathbf{x})$ and $\theta_{i}(x)$ by piecewise constant functions of the form

$$
\overline{\mathbf{C}}^{k}(\underline{\mathbf{P}}, \mathbf{x})=\sum_{j=1}^{M(k)} \chi_{\Omega_{j}^{k}} \overline{\mathbf{C}}\left(\underline{\mathbf{P}}, \mathbf{x}_{j}^{k}\right) \text { and } \theta_{i}^{k}(x)=\sum_{j=1}^{M(k)} \chi_{\Omega_{j}^{k}} \theta_{i}\left(\mathbf{x}_{j}^{k}\right) .
$$

Here $\bar{\Omega}=\bigcup_{j}^{M(k)} \bar{\Omega}_{j}$ and $\mathbf{x}_{j}^{k}$ is a point in $\Omega_{j}^{k}$. The sequence $\left\{\overline{\mathbf{C}}^{k}(\underline{\mathbf{P}}, \mathbf{x})\right\}_{k=1}^{\infty}$ converges almost everywhere to $\overline{\mathbf{C}}(\underline{\mathbf{P}}, \mathbf{x})$. This implies that the sequence $\mathrm{G}$ converges to $\overline{\mathbf{C}}(\underline{\mathbf{P}}, \mathbf{x})$; see [27]. The sequence $\left\{\theta_{i}^{k}(x)\right\}_{k=1}^{\infty}$ also converges almost everywhere to $\theta_{i}(x)$. At each sample point $\mathbf{x}_{j}^{k}$, one has

$$
\overline{\mathbf{C}}\left(\underline{\mathbf{P}}, \mathbf{x}_{j}^{k}\right) \bar{\epsilon}: \bar{\epsilon}=\int_{Q(0,1)} \overline{\mathbf{C}}_{0}\left(\underline{\mathbf{P}}, \mathbf{y}, \mathbf{x}_{j}^{k}\right)(\epsilon(\mathbf{w})+\bar{\epsilon}):(\epsilon(\mathbf{w})+\bar{\epsilon}) d \mathbf{y},
$$

where $\mathbf{w}$ is the $W_{\#}^{1,2}\left(Q(0,1), \mathbf{R}^{3}\right)$ solution of

$$
-\operatorname{div}\left(\overline{\mathbf{C}}_{0}\left(\underline{\mathbf{A}}, \mathbf{y}, \mathbf{x}_{j}^{k}\right)(\epsilon(\mathbf{w}(\mathbf{y}))+\bar{\epsilon})\right)=0 .
$$

Motivated by (2.45) and (2.46), the functions $\overline{\mathbf{C}}_{0}\left(\underline{\mathbf{A}}, \mathbf{y}, \mathbf{x}_{j}^{k}\right)$ are extended to $\mathbf{R}^{3}$ in the $\mathbf{y}$ variable by periodicity and for $k$ and $n$ sufficiently large, we define the tensor $\overline{\mathbf{C}}^{k, n}(\underline{\mathbf{P}}, \mathbf{x})$ in $\mathcal{C}(\underline{\mathbf{A}}, \mathbf{x})$ by

$$
\overline{\mathbf{C}}^{k, n}(\underline{\mathbf{P}}, \mathbf{x})=\sum_{j=1}^{M(k)} \chi_{\Omega_{j}^{k}} \overline{\mathbf{C}}_{0}\left(\underline{\mathbf{P}}, \mathbf{x} / n, \mathbf{x}_{j}^{k}\right) .
$$

From the local property of $\mathrm{G}$ convergence [27] and from the theory of periodic homogenization [3], it is evident that $\left\{\overline{\mathbf{C}}^{k, n}(\underline{\mathbf{P}}, \mathbf{x})\right\}_{n=1}^{\infty} \mathrm{G}$ converges to $\overline{\mathbf{C}}^{k}(\underline{\mathbf{P}}, \mathbf{x})$ for every $\underline{\mathbf{P}}$ in $\mathcal{N}(\underline{\mathbf{A}})$. Since the topology of $\mathrm{G}$ convergence is metrizable [8], [25], an application of the triangle inequality provides a diagonal sequence $\left\{\mathbf{C}^{k_{j}, n_{j}}(\underline{\mathbf{P}}, \mathbf{x})\right\}_{j=1}^{\infty}$ that $\mathrm{G}$ converges to $\overline{\mathbf{C}}(\underline{\mathbf{P}}, \mathbf{x})$ for every $\underline{\mathbf{P}}$ in $\mathcal{N}(\underline{\mathbf{A}})$ and the theorem follows for the case when $(\overline{\mathbf{C}}(\underline{\mathbf{A}}, \mathbf{x}), \partial \overline{\mathbf{C}}(\underline{\mathbf{A}}, \mathbf{x}))$ takes values in $S_{\underline{\theta}}(\mathbf{x})(\underline{\mathbf{A}})$.

Next given a set of functions $\theta_{i}, i=1, \ldots, N$ and $\overline{\mathbf{C}}(\underline{\mathbf{P}}, \mathbf{x})$, satisfying the hypothesis of Theorem 2.3, suppose that $(\overline{\mathbf{C}}(\underline{\mathbf{A}}, \mathbf{x}), \partial \overline{\mathbf{C}}(\underline{\mathbf{A}}, \mathbf{x}))$ takes values in the closure of $S_{\underline{\theta}(\mathbf{x})}(\underline{\mathbf{A}})$. 
Then for each $\mathbf{x}$ in $\Omega$, there is a sequence $\left\{\left(\overline{\mathbf{C}}^{j}(\underline{\mathbf{A}}, \mathbf{x}), \partial \overline{\mathbf{C}}^{j}(\underline{\mathbf{A}}, \mathbf{x})\right)\right\}_{j=1}^{\infty} \subset S_{\underline{\theta}(\mathbf{x})}(\underline{\mathbf{A}})$ that converges to $(\overline{\mathbf{C}}(\underline{\mathbf{A}}, \mathbf{x}), \partial \overrightarrow{\mathbf{C}}(\underline{\mathbf{A}}, \mathbf{x}))$. The theorem follows from similar arguments on noting that one has an approximation to $\overline{\mathbf{C}}(\underline{\mathbf{P}}, \mathbf{x})$ of the form given by $(2.44)$ and at the sample points $\overline{\mathbf{C}}\left(\underline{\mathbf{P}}, \mathbf{x}_{s}^{k}\right)=\lim _{j \rightarrow \infty} \overline{\mathbf{C}}^{j}\left(\underline{\mathbf{P}}, \mathbf{x}_{s}^{k}\right)$.

3. Homogenization of stress fluctuations and $L^{\infty}$ estimates for weak limits. In this section, a homogenization theorem is presented for quadratic functions of a weakly converging sequence of stresses associated with a $\mathrm{G}$ convergent sequence of elasticity tensors. This result is used to obtain $L^{\infty}$ estimates for the weak $L^{2}$ limit of the sequence. In what follows it is assumed that the sequence $\left\{\mathbf{C}^{n}(\underline{\mathbf{P}}, \mathbf{x})\right\}_{n=1}^{\infty} \mathrm{G}$ converges to $\mathbf{C}^{E}(\underline{\mathbf{P}}, \mathbf{x})$ for every $\underline{\mathbf{P}}$ in $\mathcal{N}(\underline{\mathbf{A}})$. Here the sequence of stresses $\left\{\sigma^{n}\right\}_{n=1}^{\infty}$ satisfy $\sigma^{n}=\mathbf{C}^{n}(\underline{\mathbf{A}}, \mathbf{x}) \epsilon\left(\mathbf{u}^{n}\right)$, where $u^{n}$ is the $W_{0}^{1,2}\left(\Omega, \mathbf{R}^{3}\right)$ solution of

$$
-\operatorname{div}\left(\mathbf{C}^{n}(\underline{\mathbf{A}}, \mathbf{x}) \epsilon\left(\mathbf{u}^{n}\right)\right)=\mathbf{f} .
$$

The weak limit of the stresses $\sigma^{M}$ is given in terms of the $W_{0}^{1,2}\left(\Omega, \mathbf{R}^{3}\right)$ solution $u^{M}$ of

$$
-\operatorname{div}\left(\mathbf{C}^{E}(\underline{\mathbf{A}}, \mathbf{x}) \epsilon\left(\mathbf{u}^{M}\right)\right)=\mathbf{f},
$$

and $\sigma^{M}=\mathbf{C}^{E}(\underline{\mathbf{A}}, \mathbf{x}) \epsilon\left(\mathbf{u}^{M}\right)$.

We begin by considering the limits of quadratic functions of sequences of weakly converging stress tensors. Given any symmetric fourth order tensor $\Pi$ and any function $p(\mathbf{x})$ in $C^{\infty}(\bar{\Omega})$, then the following homogenization result holds:

TheOREM 3.1 (Homogenization of stress fluctuations in the $i$ th phase). Given that $\left\{\mathbf{C}^{n}(\underline{\mathbf{A}}, \mathbf{x})\right\}_{n=1}^{\infty} \mathrm{G}$ converges to $\mathbf{C}^{E}(\underline{\mathbf{A}}, \mathbf{x})$, then

$$
\lim _{n \rightarrow \infty} \int_{\Omega} p(\mathbf{x}) \chi_{i}^{n}(\mathbf{x}) \Pi \sigma^{n}(\mathbf{x}): \sigma^{n}(\mathbf{x}) d \mathbf{x}=\int_{\Omega} p(\mathbf{x}) \widetilde{\Pi}_{i}(\mathbf{x}) \sigma^{M}(\mathbf{x}): \sigma^{M}(\mathbf{x}) d \mathbf{x},
$$

where

$$
\widetilde{\Pi}_{i}(\mathbf{x}) \sigma^{M}(\mathbf{x}): \sigma^{M}(\mathbf{x})=\left(\mathbf{S}^{E}(\underline{\mathbf{A}}, \mathbf{x}) \mathbf{A}_{i} \Pi \mathbf{A}_{i} \vec{\nabla}^{i} \mathbf{C}^{E}(\underline{\mathbf{A}}, \mathbf{x}) \mathbf{S}^{E}(\underline{\mathbf{A}}, \mathbf{x})\right) \sigma^{M}(\mathbf{x}): \sigma^{M}(\mathbf{x}) .
$$

Here $\mathbf{S}^{E}(\underline{\mathbf{A}}, \mathbf{x})=\left(\mathbf{C}^{E}(\underline{\mathbf{A}}, \mathbf{x})\right)^{-1}$ is the effective compliance.

Equivalently

$$
\chi_{i}^{n}(\mathbf{x}) \Pi \sigma^{n}(\mathbf{x}): \sigma^{n}(\mathbf{x}) \rightarrow\left(\mathbf{S}^{E}(\underline{\mathbf{A}}, \mathbf{x}) \mathbf{A}_{i} \Pi \mathbf{A}_{i} \vec{\nabla}^{i} \mathbf{C}^{E}(\underline{\mathbf{A}}, \mathbf{x}) \mathbf{S}^{E}(\underline{\mathbf{A}}, \mathbf{x})\right) \sigma^{M}(\mathbf{x}): \sigma^{M}(\mathbf{x})
$$

in the sense of distributions. We specialize to the case $\Pi=\mathbf{I}$ where $\mathbf{I}$ is the fourth order identity tensor; then $\Pi \sigma^{n}: \sigma^{n}=\left|\sigma^{n}\right|^{2}$ and we write

$$
\begin{aligned}
\lim _{n \rightarrow \infty} \int_{\Omega} p(\mathbf{x})\left|\sigma^{n}\right|^{2} d \mathbf{x}= & \lim _{n \rightarrow \infty} \int_{\Omega} p(\mathbf{x})\left|\sigma^{n}-\sigma^{M}\right|^{2} d \mathbf{x} \\
& +\int_{\Omega} p(\mathbf{x})\left|\sigma^{M}\right|^{2} d \mathbf{x} .
\end{aligned}
$$

From Theorem 3.1 it follows that

$$
\left|\sigma^{n}(\mathbf{x})-\sigma^{M}(\mathbf{x})\right|^{2} \rightarrow \mathbf{Q}(\mathbf{x}) \sigma^{M}(\mathbf{x}): \sigma^{M}(\mathbf{x})
$$


in the sense of distributions, where $\mathbf{Q}$ is given by

$$
\mathbf{Q}(\mathbf{x})=\left\{\sum_{i=1}^{N}\left(\mathbf{S}^{E}(\underline{\mathbf{A}}, \mathbf{x}) \mathbf{A}_{i}^{2} \vec{\nabla}^{i} \mathbf{C}^{E}(\underline{\mathbf{A}}, \mathbf{x}) \mathbf{S}^{E}(\underline{\mathbf{A}}, \mathbf{x})\right\}-\mathbf{I} .\right.
$$

From (3.7) it is evident that the tensor $\mathbf{Q}$ is positive definite. The next theorem provides an upper bound on the $L^{\infty}$ norm for the homogenized stress $\sigma^{M}$.

Theorem 3.2. Given that $\left\{\mathbf{C}^{n}(\underline{\mathbf{A}}, \mathbf{x})\right\}_{n=1}^{\infty} \mathrm{G}$ converges to $\mathbf{C}^{E}(\underline{\mathbf{A}}, \mathbf{x})$, with $\sigma^{n}=$ $\mathbf{C}^{n}(\underline{\mathbf{A}}, \mathbf{x}) \epsilon\left(\mathbf{u}^{n}\right)$ and $\sigma^{M}=\mathbf{C}^{E}(\underline{\mathbf{A}}, \mathbf{x}) \epsilon\left(\mathbf{u}^{M}\right)$, then

$$
\left\{(\mathbf{I}+\mathbf{Q}(\mathbf{x})) \sigma^{M}(\mathbf{x}): \sigma^{M}(\mathbf{x})\right\} \leq \liminf _{n \rightarrow \infty}\left\|\left|\sigma^{n}\right|^{2}\right\|_{\infty},
$$

almost everywhere.

The homogenized versions of the stress constraints (1.7) and (1.10) follow from Theorems 3.1 and 3.2 and are given in the following corollaries.

Corollary 3.1 . If for a collection of nonnegative functions $\left\{p_{j}\right\}_{j=1}^{L}$ in $C^{\infty}(\bar{\Omega})$, it is known that

$$
\int_{\Omega} p_{j}(\mathbf{x})\left|\sigma^{n}\right|^{2} d \mathbf{x} \leq K^{2} \int_{\Omega} p_{j}(\mathbf{x}) d \mathbf{x}, \text { for every } n,
$$

then $\sigma^{M}$ satisfies

$$
\int_{\Omega} p_{j}(\mathbf{x})(\mathbf{I}+\mathbf{Q}(\mathbf{x})) \sigma^{M}(\mathbf{x}): \sigma^{M}(\mathbf{x}) d \mathbf{x} \leq K^{2} \int_{\Omega} p_{j}(\mathbf{x}) d \mathbf{x} .
$$

Corollary 3.2. If it is known that

$$
\left\|\left|\sigma^{n}\right|\right\|_{\infty} \leq K, \text { for every } n,
$$

then $\sigma^{M}$ satisfies

$$
\sqrt{(\mathbf{I}+\mathbf{Q}(\mathbf{x})) \sigma^{M}(\mathbf{x}): \sigma^{M}(\mathbf{x})} \leq K
$$

almost everywhere.

3.1. The local homogenization theorem for stress and strain fluctuations. In this subsection the local homogenization Theorem 3.3 is established. The local homogenization theorem is applied in the following subsection to establish Theorem 3.1. Consider a sequence $\left\{\mathbf{C}^{n}(\underline{\mathbf{P}}, \mathbf{x})\right\}_{n=1}^{\infty}$ that $\mathrm{G}$ converges to $\mathbf{C}^{E}(\underline{\mathbf{P}}, \mathbf{x})$ for every $\underline{\mathbf{P}}$ in $\mathcal{N}(\underline{\mathbf{A}})$. Given a constant strain $\bar{\epsilon}$ and an open subset $\omega$ of $\Omega$, the local corrector function $\mathbf{w}_{\bar{\epsilon}}^{n}$ is the $W_{0}^{1,2}\left(\omega ; \mathbf{R}^{3}\right)$ solution of

$$
-\operatorname{div}\left(\mathbf{C}^{n}(\underline{\mathbf{A}} \mathbf{x})\right)\left(\epsilon\left(\mathbf{w}_{\bar{\epsilon}}^{n}\right)+\bar{\epsilon}\right)=-\operatorname{div}\left(\mathbf{C}^{E}(\underline{\mathbf{A}}, \mathbf{x}) \bar{\epsilon}\right) \text {, in } \omega .
$$

The differential equation (3.14) is written in the weak form given by

$$
\int_{\omega} \mathbf{C}^{n}(\underline{\mathbf{A}}, \mathbf{x})\left(\epsilon\left(\mathbf{w}_{\bar{\epsilon}}^{n}\right)+\bar{\epsilon}\right): \epsilon(\mathbf{v}) d \mathbf{x}-\int_{\omega} \mathbf{C}^{E}(\underline{\mathbf{A}}, \mathbf{x}) \bar{\epsilon}: \epsilon(\mathbf{v}) d \mathbf{x}=0,
$$

for all $\mathbf{v}$ in $W_{0}^{1,2}\left(\omega ; \mathbf{R}^{3}\right)$. In the sequel, a generic open subset of $\Omega$ is denoted by $\omega$. The volume of $\omega$ is denoted by $|\omega|$ and an application of Cauchy's inequality in (3.15) give

$$
\int_{\omega}\left|\epsilon\left(\mathbf{w}_{\bar{\epsilon}}^{n}\right)\right|^{2} d \mathbf{x} \leq\left(4 \Lambda^{2} / \lambda^{2}\right)|\omega||\bar{\epsilon}|^{2}
$$


and

$$
\int_{\omega}\left|\epsilon\left(\mathbf{w}_{\bar{\epsilon}}^{n}\right)+\bar{\epsilon}\right|^{2} d \mathbf{x} \leq\left(4 \Lambda^{2} / \lambda^{2}+1\right)|\omega||\bar{\epsilon}|^{2} .
$$

Integration by parts and taking limits shows that sequence of corrector functions converge weakly to the following limits given by

Property 3.1. The sequence $\left\{\mathbf{w}_{\bar{\epsilon}}^{n}\right\}_{n=1}^{\infty}$ converges weakly to zero in $W_{0}^{1,2}\left(\omega ; \mathbf{R}^{3}\right) ;$ thus

$$
\epsilon\left(\mathbf{w}_{\bar{\epsilon}}^{n}\right) \rightarrow 0
$$

and

$$
\mathbf{C}^{n}(\underline{\mathbf{A}}, \mathbf{x})\left(\epsilon\left(\mathbf{w}_{\bar{\epsilon}}^{n}\right)+\bar{\epsilon}\right) \rightarrow \mathbf{C}^{E}(\underline{\mathbf{A}}, \mathbf{x}) \bar{\epsilon} .
$$

Moreover, passing to a subsequence, if necessary, standard results show that $\lim _{n \rightarrow \infty} \int_{\omega}\left|\mathbf{w}_{\bar{\epsilon}}^{n}\right|^{2} d \mathbf{x}=0$. It is Property 3.1 that makes the correctors useful for our purposes.

We set $\delta \mathbf{C}=\mathbf{C}^{E}(\underline{\mathbf{A}}+\delta \beta \underline{\mathbf{M}}, \mathbf{x})-C^{E}(\underline{\mathbf{A}}, \mathbf{x})$ and examine the dependence of $(1 /|\omega|) \int_{\omega} \delta \mathbf{C} \bar{\epsilon}: \bar{\epsilon} d \mathbf{x}$ with respect to the increment $\delta \beta$. The correctors associated with the sequence $\left\{\mathbf{C}^{n}(\underline{\mathbf{A}}+\delta \beta \underline{\mathbf{M}}, \mathbf{x})\right\}_{n=1}^{\infty}$ are denoted by $\hat{\mathbf{w}}_{\bar{\epsilon}}^{n}+\bar{\epsilon} \mathbf{x}$, where $\hat{\mathbf{w}}_{\bar{\epsilon}}^{n}$ are $W_{0}^{1,2}\left(\omega ; \mathbf{R}^{3}\right)$ solutions of

$$
\int_{\omega} \mathbf{C}^{n}(\underline{\mathbf{A}}+\delta \beta \underline{\mathbf{M}}, \mathbf{x})\left(\epsilon\left(\hat{\mathbf{w}}_{\bar{\epsilon}}^{n}\right)+\bar{\epsilon}\right): \epsilon(\mathbf{v}) d \mathbf{x}-\int_{\omega} \mathbf{C}^{E}(\underline{\mathbf{A}}+\delta \beta \underline{\mathbf{M}}, \mathbf{x}) \bar{\epsilon}: \epsilon(\mathbf{v}) d \mathbf{x}=0,
$$

for all $\mathbf{v}$ in $W_{0}^{1,2}\left(\omega ; \mathbf{R}^{3}\right)$ and

$$
\begin{aligned}
\mathbf{C}^{n}(\underline{\mathbf{A}}+\delta \beta \underline{\mathbf{M}}, \mathbf{x})\left(\epsilon\left(\hat{\mathbf{w}}_{\bar{\epsilon}}^{n}\right)+\bar{\epsilon}\right) & \rightarrow C^{E}(\underline{\mathbf{A}}+\delta \beta \underline{\mathbf{M}}, \mathbf{x}) \bar{\epsilon} \\
\epsilon\left(\hat{\mathbf{w}}_{\bar{\epsilon}}^{n}\right) & \rightarrow 0 .
\end{aligned}
$$

For any choice of test function $p(x)$ that is differentiable on $\omega$ and continuous on $\bar{\omega}$, it follows from (3.19) and (3.21) that

$$
\begin{aligned}
(1 /|\omega|) \int_{\omega} p(x) \delta \mathbf{C} \bar{\epsilon}: \bar{\epsilon} d \mathbf{x}= & \lim _{n \rightarrow \infty}(1 /|\omega|) \int_{\omega} p(x) \mathbf{C}^{n}(\underline{\mathbf{A}}+\delta \beta \underline{\mathbf{M}}, \mathbf{x})\left(\epsilon\left(\hat{\mathbf{w}}_{\bar{\epsilon}}^{n}\right)+\bar{\epsilon}\right): \bar{\epsilon} d \mathbf{x} \\
& -\lim _{n \rightarrow \infty}(1 /|\omega|) \int_{\omega} p(x) \mathbf{C}^{n}(\underline{\mathbf{A}}, \mathbf{x})\left(\epsilon\left(\mathbf{w}_{\bar{\epsilon}}^{n}\right)+\bar{\epsilon}\right): \bar{\epsilon} d \mathbf{x} .
\end{aligned}
$$

Writing $\mathbf{C}^{n}(\underline{\mathbf{A}}+\delta \beta \underline{\mathbf{M}}, \mathbf{x})=\mathbf{C}^{n}(\underline{\mathbf{A}}, \mathbf{x})+\delta \beta \mathbf{M}_{i} \chi_{i}^{n}$ and $\hat{\mathbf{w}}_{\bar{\epsilon}}^{n}=\mathbf{w}_{\bar{\epsilon}}^{n}+\delta \mathbf{w}^{n}$, where $\delta \mathbf{w}^{n}=$ $\hat{\mathbf{w}}_{\bar{\epsilon}}^{n}-\mathbf{w}_{\bar{\epsilon}}^{n}$ and substitution into (3.23) gives

$$
\begin{aligned}
& (1 /|\omega|) \int_{\omega} p \delta \mathbf{C} \bar{\epsilon}: \bar{\epsilon} d \mathbf{x}=\lim _{n \rightarrow \infty} \\
& \times(1 /|\omega|) \int_{\omega} p \delta \beta \mathbf{M}_{i} \chi_{i}^{n}\left(\epsilon\left(\mathbf{w}_{\bar{\epsilon}}^{n}\right)+\bar{\epsilon}\right): \bar{\epsilon}+p \delta \beta \mathbf{M}_{i} \chi_{i}^{n} \epsilon\left(\delta \mathbf{w}^{n}\right): \bar{\epsilon}+p \mathbf{C}^{n}(\underline{\mathbf{A}}, \mathbf{x}) \epsilon\left(\delta \mathbf{w}^{n}\right): \bar{\epsilon} d \mathbf{x} .
\end{aligned}
$$

To proceed further, subtract (3.15) from (3.20) to obtain for every $\mathbf{v}$ in $W_{0}^{1,2}\left(\omega ; \mathbf{R}^{3}\right)$ the equation

$$
\begin{aligned}
0= & \int_{\omega} \delta \beta \mathbf{M}_{i} \chi_{i}^{n}\left(\epsilon\left(\mathbf{w}_{\bar{\epsilon}}^{n}\right)+\bar{\epsilon}\right): \epsilon(\mathbf{v}) d \mathbf{x}+\int_{\omega} \mathbf{C}^{n}(\underline{\mathbf{A}}, \mathbf{x}) \epsilon\left(\delta \mathbf{w}^{n}\right): \epsilon(\mathbf{v}) d \mathbf{x} \\
& +\int_{\omega} \delta \beta \mathbf{M}_{i} \chi_{i}^{n} \epsilon\left(\delta \mathbf{w}^{n}\right): \epsilon(\mathbf{v}) d \mathbf{x}-\int_{\omega} \delta \mathbf{C} \bar{\epsilon}: \epsilon(\mathbf{v}) d \mathbf{x} .
\end{aligned}
$$


Choosing $\mathbf{v}=\left(p \mathbf{w}_{\bar{\epsilon}}^{n}\right)$ in (3.25), substitution into (3.24), and taking limits gives

$(1 /|\omega|) \int_{\omega} p \delta \mathbf{C} \bar{\epsilon}: \bar{\epsilon} d \mathbf{x}=\delta \beta \lim _{n \rightarrow \infty}$

$\times\left((1 /|\omega|) \int_{\omega} p \mathbf{M}_{i} \chi_{i}^{n}\left(\epsilon\left(\mathbf{w}_{\bar{\epsilon}}^{n}\right)+\bar{\epsilon}\right):\left(\epsilon\left(\mathbf{w}_{\bar{\epsilon}}^{n}\right)+\bar{\epsilon}\right) d \mathbf{x}+(1 /|\omega|) \int_{\omega} p \mathbf{M}_{i} \chi_{i}^{n}\left(\epsilon\left(\mathbf{w}_{\bar{\epsilon}}^{n}\right)+\bar{\epsilon}\right): \epsilon\left(\delta \mathbf{w}^{n}\right) d \mathbf{x}\right)$.

Writing

$$
\begin{aligned}
& \overline{\mathcal{S}}=\limsup _{n \rightarrow \infty}(1 /|\omega|) \int_{\omega} p \delta \beta \mathbf{M}_{i} \chi_{i}^{n}\left(\epsilon\left(\mathbf{w}_{\bar{\epsilon}}^{n}\right)+\bar{\epsilon}\right): \epsilon\left(\delta \mathbf{w}^{n}\right) d \mathbf{x} \text { and } \\
& \underline{\mathcal{S}}=\liminf _{n \rightarrow \infty}(1 /|\omega|) \int_{\omega} p \delta \beta \mathbf{M}_{i} \chi_{i}^{n}\left(\epsilon\left(\mathbf{w}_{\bar{\epsilon}}^{n}\right)+\bar{\epsilon}\right): \epsilon\left(\delta \mathbf{w}^{n}\right) d \mathbf{x},
\end{aligned}
$$

an application of Cauchy's inequality gives

$$
|\overline{\mathcal{S}}| \leq\|p\|_{\infty} K|\bar{\epsilon}|^{2} \sqrt{|\delta \beta|} \text { and }|\underline{\mathcal{S}}| \leq\|p\|_{\infty} K|\bar{\epsilon}|^{2} \sqrt{|\delta \beta|},
$$

where $K$ is a constant independent of $\delta \beta$ and choice of $\omega \subset \Omega$ and $\|p\|_{\infty}$ is the maximum value of $p$ on $\bar{\omega}$. From this one arrives at the

Property 3.2. For every $\omega \subset \Omega$ and differentiable function $p$ continuous on $\bar{\omega}$,

$$
\begin{aligned}
(1 /|\omega|) \int_{\omega} p \delta \mathbf{C} \bar{\epsilon}: \bar{\epsilon} d \mathbf{x}= & \delta \beta \lim _{n \rightarrow \infty}\left((1 /|\omega|) \int_{\omega} p \mathbf{M}_{i} \chi_{i}^{n}\left(\epsilon\left(\mathbf{w}_{\bar{\epsilon}}^{n}\right)+\bar{\epsilon}\right):\left(\epsilon\left(\mathbf{w}_{\bar{\epsilon}}^{n}\right)+\bar{\epsilon}\right) d \mathbf{x}\right) \\
& +\delta \beta R(\delta \beta, \omega, p),
\end{aligned}
$$

with

$$
R(\delta \beta, \omega, p)=\lim _{n \rightarrow \infty}\left((1 /|\omega|) \int_{\omega} p \mathbf{M}_{i} \chi_{i}^{n}\left(\epsilon\left(\mathbf{w}_{\bar{\epsilon}}^{n}\right)+\bar{\epsilon}\right): \epsilon\left(\delta \mathbf{w}^{n}\right) d \mathbf{x}\right)
$$

and

$$
|R(\delta \beta, \omega, p)| \leq\|p\|_{\infty} K|\bar{\epsilon}|^{2} \sqrt{|\delta \beta|} .
$$

Next consider subsets of $\Omega$ given by cubes $Q(\mathbf{x}, r)$ and the associated local corrector functions are denoted by $\mathbf{w}_{\bar{\epsilon}}^{n, r}$. Put

$$
H(\mathbf{x}, r)=\lim _{n \rightarrow \infty}(1 /|Q(\mathbf{x}, r)|) \int_{Q(\mathbf{x}, r)} \mathbf{M}_{i} \chi_{i}^{n}\left(\epsilon\left(\mathbf{w}_{\bar{\epsilon}}^{n, r}\right)+\bar{\epsilon}\right):\left(\epsilon\left(\mathbf{w}_{\bar{\epsilon}}^{n, r}\right)+\bar{\epsilon}\right) d \mathbf{y} .
$$

Given the increment $\delta \beta$ consider the intersection of Lebesgue points of $\mathbf{C}^{E}(\underline{\mathbf{A}}, \mathbf{x})$ and $\mathbf{C}^{E}(\underline{\mathbf{A}}+\delta \beta \underline{\mathbf{M}}, \mathbf{x})$. Choose $p=1$ and from Property 3.2 one has

$$
\begin{aligned}
\delta \mathbf{C} \bar{\epsilon}: \bar{\epsilon} & =\lim _{r \rightarrow 0}(1 /|Q(\mathbf{x}, r)|) \int_{Q(\mathbf{x}, r)} \delta \mathbf{C} \bar{\epsilon}: \bar{\epsilon} d \mathbf{y} \\
& =\delta \beta \lim _{r \rightarrow 0}(H(\mathbf{x}, r)+R(\delta \beta, \mathbf{x})),
\end{aligned}
$$

almost everywhere on $\Omega$ and for any constant strain $\bar{\epsilon}$. Here $|R(\delta \beta, \mathbf{x})|<K|\bar{\epsilon}|^{2} \sqrt{|\delta \beta|}$. It is evident from (3.33) that $\frac{\partial \mathbf{C}^{E}}{\partial \mathbf{M}_{i}} \bar{\epsilon}: \bar{\epsilon}$ is also given by

$$
\frac{\partial \mathbf{C}^{E}}{\partial \mathbf{M}_{i}} \bar{\epsilon}: \bar{\epsilon}=\lim _{r \rightarrow 0} \lim _{n \rightarrow \infty}(1 /|Q(\mathbf{x}, r)|) \int_{Q(\mathbf{x}, r)} \mathbf{M}_{i} \chi_{i}^{n}\left(\epsilon\left(\mathbf{w}_{\bar{\epsilon}}^{n, r}\right)+\bar{\epsilon}\right):\left(\epsilon\left(\mathbf{w}_{\bar{\epsilon}}^{n, r}\right)+\bar{\epsilon}\right) d \mathbf{y} .
$$


Collecting results, one substitutes (3.33) into (3.29) of Property 3.2 to obtain the local homogenization result.

TheOREm 3.3 (Local Homogenization). Given any $\omega \subset \Omega$ and any differentiable test function $p$ continuous on $\bar{\omega}$, the sequence of local correctors, $\left\{\mathbf{w}_{\bar{\epsilon}}^{n}+\bar{\epsilon} \mathbf{x}\right\}_{n=1}^{\infty}$ defined by (3.14), satisfy

$$
\begin{aligned}
\lim _{n \rightarrow \infty}(1 /|\omega|) \int_{\omega} p \mathbf{M}_{i} \chi_{i}^{n}\left(\epsilon\left(\mathbf{w}_{\bar{\epsilon}}^{n}\right)+\bar{\epsilon}\right):\left(\epsilon\left(\mathbf{w}_{\bar{\epsilon}}^{n}\right)+\bar{\epsilon}\right) d \mathbf{x} & =(1 /|\omega|) \int_{\omega} p \frac{\partial \mathbf{C}^{E}}{\partial \mathbf{M}_{i}} \bar{\epsilon}: \bar{\epsilon} d \mathbf{x} \\
& =(1 /|\omega|) \int_{\omega} p \mathbf{M}_{i} \vec{\nabla}^{i} \mathbf{C}^{E} \bar{\epsilon}: \bar{\epsilon} d \mathbf{x}
\end{aligned}
$$

For future reference it is noted that the estimate (3.17) gives the bound

$$
\left(\vec{\nabla}^{i} \mathbf{C}^{E}(\underline{\mathbf{A}}, \mathbf{x})\right) \bar{\epsilon}: \bar{\epsilon} \leq\left(4 \Lambda^{2} / \lambda^{2}+1\right)|\bar{\epsilon}|^{2},
$$

almost everywhere in $\Omega$.

3.2. Homogenization of stress fluctuations. In this subsection Theorem 3.3 is used to establish Theorem 3.1. Consider a sequence of elasticity tensors $\left\{\mathbf{C}^{n}(\underline{\mathbf{P}}, \mathbf{x})\right\}_{n=1}^{\infty}$ that $\mathrm{G}$ converges to $C^{E}(\underline{\mathbf{P}}, \mathbf{x})$ for every $\underline{\mathbf{P}}$ in $\mathcal{N}(\underline{\mathbf{A}})$. Here $\mathbf{u}^{n}$ is the $W_{0}^{1,2}\left(\Omega ; \mathbf{R}^{3}\right)$ solution of

$$
-\operatorname{div}\left(\mathbf{C}^{n}(\underline{\mathbf{A}}, \mathbf{x}) \epsilon\left(\mathbf{u}^{n}\right)\right)=\mathbf{f}
$$

and $\mathbf{u}^{M}$ is the $W_{0}^{1,2}\left(\Omega ; \mathbf{R}^{3}\right)$ solution of

$$
-\operatorname{div}\left(\mathbf{C}^{E}(\underline{\mathbf{A}}, \mathbf{x}) \epsilon\left(\mathbf{u}^{M}\right)\right)=\mathbf{f},
$$

where $\mathbf{u}^{n}$ converges weakly to $\mathbf{u}^{M}$ in $W_{0}^{1,2}\left(\Omega ; \mathbf{R}^{3}\right)$ as $n$ tends to infinity. The first step is to approximate $\mathbf{u}^{M}$ by piecewise affine functions. Given $\delta>0$ there exists a function $\mathbf{w}^{\delta}$ in $W_{0}^{1,2}\left(\Omega ; \mathbf{R}^{3}\right)$ which is piecewise affine on $\Omega$ and

$$
\int_{\Omega}\left|\epsilon\left(\mathbf{w}^{\delta}\right)-\epsilon\left(\mathbf{u}^{M}\right)\right|^{2} d \mathbf{x}<\delta^{2}
$$

see, for example, [7]. The strain $\epsilon\left(\mathbf{w}^{\delta}\right)$ is constant on the open sets $\omega_{\delta}^{i}$ and $\bar{\Omega}=\bigcup_{i=1}^{\kappa(\delta)} \bar{\omega}_{\delta}^{i}$. In each open set $\omega_{\delta}^{i}$, one has $\epsilon\left(\mathbf{w}^{\delta}\right)=\bar{\epsilon}^{i} \mathbf{x}+c^{i}$, where $\bar{\epsilon}^{i}$ is a constant strain and $c^{i}$ is a constant vector.

The right-hand side $f^{\delta}=-\operatorname{div}\left(\mathbf{C}^{E}(\underline{\mathbf{A}}, \mathbf{x}) \epsilon\left(\mathbf{w}^{\delta}\right)\right)$ is chosen and $\mathbf{w}^{n, \delta}$ is defined to be the $W_{0}^{1,2}\left(\Omega ; \mathbf{R}^{3}\right)$ solution of

$$
-\operatorname{div}\left(\mathbf{C}^{n}(\underline{\mathbf{A}}, \mathbf{x}) \epsilon\left(\mathbf{w}^{\varepsilon, \delta}\right)\right)=f^{\delta} .
$$

Integration by parts and taking limits shows that $\mathbf{w}^{n, \delta}$ converges weakly to $\mathbf{w}^{\delta}$ in $W_{0}^{1,2}\left(\Omega ; \mathbf{R}^{3}\right)$ as $n$ goes to infinity and

$$
\mathbf{C}^{n}(\underline{\mathbf{A}}, \mathbf{x}) \epsilon\left(\mathbf{w}^{n, \delta}\right) \rightarrow \mathbf{C}^{E}(\underline{\mathbf{A}}, \mathbf{x}) \epsilon\left(\mathbf{w}^{\delta}\right) .
$$

The error between the weakly converging sequences $\left\{\mathbf{u}^{n}\right\}_{n=1}^{\infty}$ and the sequence of global corrector functions $\left\{\mathbf{w}^{n, \delta}\right\}_{n=1}^{\infty}$ can be controlled uniformly with respect to $n$ : Indeed, application of Cauchy's inequality gives a constant $C_{0}$ independent of $n$ and $\delta$ such that

$$
\int_{\Omega}\left|\epsilon\left(\mathbf{u}^{n}\right)-\epsilon\left(\mathbf{w}^{n, \delta}\right)\right|^{2} d \mathbf{x} \leq C_{0}^{2}\left(\frac{\Lambda}{\lambda}\right)^{2} \delta^{2}, \text { for all } n>0 .
$$


Collecting results we write $\mathbf{u}=\mathbf{w}^{\delta}+\mathbf{r}^{\delta}, \mathbf{u}^{n}=\mathbf{w}^{n, \delta}+\mathbf{z}^{n, \delta}$. Here $\mathbf{r}^{\delta}$ and $\mathbf{z}^{n, \delta}$ are in $W_{0}^{1,2}\left(\Omega ; \mathbf{R}^{3}\right)$ and satisfy

$$
\int_{\Omega}\left|\epsilon\left(\mathbf{r}^{\delta}\right)\right|^{2} d \mathbf{x}<\delta^{2} \text { and } \int_{\Omega}\left|\epsilon\left(\mathbf{z}^{n, \delta}\right)\right|^{2} d \mathbf{x}<\delta^{2}, \text { for all } n>0 .
$$

Given a differentiable test function $p(\mathbf{x})$ continuous on $\bar{\Omega}$, one writes

$$
\begin{aligned}
\int_{\Omega} & p \chi_{j}^{n} \Pi \sigma^{n}: \sigma^{n} d \mathbf{x} \\
& =\int_{\Omega} p \chi_{j}^{n} \Pi \mathbf{A}_{j}\left(\epsilon\left(\mathbf{w}^{n, \delta}\right)+\epsilon\left(\mathbf{z}^{n, \delta}\right)\right): \mathbf{A}_{j}\left(\epsilon\left(\mathbf{w}^{n, \delta}\right)+\epsilon\left(\mathbf{z}^{n, \delta}\right)\right) d \mathbf{x} \\
& =\sum_{i=1}^{\kappa(\delta)} \int_{\omega_{\delta}^{i}} p \chi_{j}^{n} \Pi \mathbf{A}_{j} \epsilon\left(\mathbf{w}^{n, \delta}\right): \mathbf{A}_{j} \epsilon\left(\mathbf{w}^{n, \delta}\right) d \mathbf{x}+O(\delta) .
\end{aligned}
$$

The following observation follows easily from integration by parts and the definitions of $\mathrm{G}$ convergence.

LoCAlization Property. Let $\mathbf{w}_{\bar{\epsilon}^{i}}^{i, n}$ be the solution of

$$
-\operatorname{div}\left(\mathbf{C}^{n}(\underline{\mathbf{A}}, \mathbf{x})\right)\left(\epsilon\left(\mathbf{w}_{\bar{\epsilon}^{i}}^{i, n, \delta}\right)+\bar{\epsilon}^{i}\right)=-\operatorname{div}\left(\mathbf{C}^{E}(\underline{\mathbf{A}}, \mathbf{x}) \bar{\epsilon}^{i}\right)
$$

on $\omega_{\delta}^{i}$, where $\mathbf{w}_{\bar{\epsilon}^{i}}^{i, n, \delta}=0$ on the boundary of $\omega_{\delta}^{i}$; then

$$
\lim _{n \rightarrow \infty} \int_{\omega_{\delta}^{i}}\left|\epsilon\left(\mathbf{w}_{\bar{\epsilon}^{i}}^{i, n, \delta}\right)+\bar{\epsilon}^{i}-\epsilon\left(\mathbf{w}^{n, \delta}\right)\right|^{2} d \mathbf{x}=0 .
$$

Applying Theorem 3.3 and working up from small to intermediate scales, one finds that

$$
\begin{aligned}
& \sum_{i=1}^{\kappa(\delta)} \int_{\omega_{\delta}^{i}} p \mathbf{A}_{j} \Pi \mathbf{A}_{j} \nabla^{j} \mathbf{C}^{E}(\underline{\mathbf{A}}, \mathbf{x}) \bar{\epsilon}^{i}: \bar{\epsilon}^{i} d \mathbf{x} \\
& =\sum_{i=1}^{\kappa(\delta)} \lim _{n \rightarrow \infty} \int_{\omega_{\delta}^{i}} p \chi_{j}^{n} \Pi \mathbf{A}_{j}\left(\epsilon\left(\mathbf{w}_{\bar{\epsilon}^{i}}^{i, n, \delta}\right)+\bar{\epsilon}^{i}\right): \mathbf{A}_{j}\left(\epsilon\left(\mathbf{w}_{\bar{\epsilon}^{i}}^{i, n, \delta}\right)+\bar{\epsilon}^{i}\right) d \mathbf{x} \\
& =\lim _{n \rightarrow \infty} \sum_{i=1}^{\kappa(\delta)} \int_{\omega_{\delta}^{i}} p \chi_{j}^{n} \Pi \mathbf{A}_{j} \epsilon\left(\mathbf{w}^{n, \delta}\right): \mathbf{A}_{j} \epsilon\left(\mathbf{w}^{n, \delta}\right) d \mathbf{x} .
\end{aligned}
$$

Proceeding from large scales down to intermediate scales, we see from (3.36), (3.39), (3.44), and (3.47) that

$$
\begin{aligned}
\lim _{n \rightarrow \infty} \int_{\Omega} p \chi_{j}^{n} \Pi \sigma^{n}: \sigma^{n} d \mathbf{x} & =\sum_{i=1}^{\kappa(\delta)} \int_{\omega_{\delta}^{i}} p \mathbf{A}_{j} \Pi \mathbf{A}_{j} \vec{\nabla}^{j} \mathbf{C}^{E}(\underline{\mathbf{A}}, \mathbf{x}) \bar{\epsilon}^{i}: \bar{\epsilon}^{i} d \mathbf{x}+O(\delta) \\
& =\int_{\Omega} p \mathbf{A}_{j} \Pi \mathbf{A}_{j} \vec{\nabla}^{j} \mathbf{C}^{E}(\underline{\mathbf{A}}, \mathbf{x}) \epsilon\left(\mathbf{u}^{M}\right): \epsilon\left(\mathbf{u}^{M}\right) d \mathbf{x}+O(\delta) \\
& =\int_{\Omega} p \mathbf{A}_{j} \Pi \mathbf{A}_{j} \vec{\nabla}^{j} \mathbf{C}^{E}(\underline{\mathbf{A}}, \mathbf{x}) \mathbf{S}^{E}(\underline{\mathbf{A}}, \mathbf{x}) \sigma^{M}: \mathbf{S}^{E}(\underline{\mathbf{A}}, \mathbf{x}) \sigma^{M} d \mathbf{x}+O(\delta)
\end{aligned}
$$

and Theorem 3.1 follows noting that $\delta$ is arbitrary. 
To establish Theorem 3.2, note that for any positive test function $p$ in $C^{\infty}(\bar{\Omega})$, Hölder's inequality gives

$$
\int_{\Omega} p(\mathbf{x})\left(\left|\sigma^{n}\right|^{2}-\left\|\left|\sigma^{n}\right|^{2}\right\|_{\infty}\right) d \mathbf{x} \leq 0
$$

Sending $n$ to infinity together with Theorem 3.1 gives

$$
\int_{\Omega} p(\mathbf{x})\left((\mathbf{I}+\mathbf{Q}) \sigma^{M}: \sigma^{M}-\liminf _{n \rightarrow \infty}\left\|\left|\sigma^{n}\right|^{2}\right\|_{\infty}\right) d \mathbf{x} \leq 0,
$$

for every choice of $p$ and Theorem 3.2 follows.

4. The stress constrained $G$ closure and relaxation of stress constrained design problems. The relaxed formulation of the design problems $P^{L}$ and $P^{\infty}$ is introduced using the notion of stress constrained $\mathrm{G}$ closure. To do this we reformulate the design problems $P^{L}$ and $P^{\infty}$ in an equivalent way. For a given tolerance $K$, we define the set of controls denoted by $\mathcal{A} d^{\infty}(K, \underline{\mathbf{A}}, \underline{\gamma})$ to be the set of all local elasticity tensors $\mathbf{C}(\underline{\mathbf{P}}, \mathbf{x})=\sum_{i=1}^{N} \mathbf{P}_{i} \chi_{i}(\mathbf{x})$ with $\underline{\mathbf{P}}$ in $\mathcal{N}(\underline{\mathbf{A}}), \int_{\Omega} \chi_{i} d \mathbf{x} \leq \gamma_{i}$, such that

$$
\||\sigma|\|_{\infty} \leq K
$$

where $\sigma(\mathbf{x})=\mathbf{C}(\underline{\mathbf{A}}, \mathbf{x}) \epsilon(\mathbf{u}(\mathbf{x}))$, and $\mathbf{u}$ is the $W_{0}^{1,2}\left(\Omega, \mathbf{R}^{3}\right)$ solution of

$$
-\operatorname{div}(\mathbf{C}(\underline{\mathbf{A}}, \mathbf{x}) \epsilon(\mathbf{u}))=\mathbf{f} .
$$

The $P^{\infty}$ design problem is given by

$$
P^{\infty}=\inf _{\mathcal{A} d^{\infty}(K, \underline{\mathbf{A}} \cdot \underline{\gamma})} F(\mathbf{u}) .
$$

Recent developments in regularity theory [2], [11], [12], imply that the class of controls $\mathcal{A} d^{\infty}(K, \underline{\mathbf{A}}, \underline{\gamma})$ is generated by a strikingly large class of configurations. The set of controls denoted by $\mathcal{A} d^{L}(K, \underline{\mathbf{A}}, \underline{\gamma})$ is defined to be all local elasticity tensors $\mathbf{C}(\underline{\mathbf{P}}, \mathbf{x})=$ $\sum_{i=1}^{N} \mathbf{P}_{i} \chi_{i}(\mathbf{x})$ with $\underline{\mathbf{P}}$ in $\mathcal{N}(\underline{\mathbf{A}}), \int_{\Omega} \chi_{i} d \mathbf{x} \leq \gamma_{i}$ associated with solutions $\mathbf{u}$ of $(4.2)$ satisfying the finite number of integral stress constraints given by (1.10). The $P^{L}$ design problem is given by

$$
P^{L}=\inf _{\mathcal{A} d^{L}(K, \underline{\mathbf{A}}, \underline{\gamma})} F(\mathbf{u})
$$

The $\mathrm{G}$ closure of $\mathcal{A} d^{\infty}(K, \underline{\mathbf{A}}, \underline{\gamma})$ is defined to be all functions $\left(\widetilde{\mathbf{C}}(\underline{\mathbf{P}}, \mathbf{x}), \theta_{1}(\mathbf{x}), \ldots, \theta_{N}(\mathbf{x})\right)$ for which there exist sequences $\left\{\mathbf{C}^{n}(\underline{\mathbf{P}}, \mathbf{x})\right\}_{i=1}^{n}$ in $\mathcal{A} d^{\infty}(K, \underline{\mathbf{A}}, \underline{\gamma}) \mathrm{G}$ converging in $\widetilde{\mathbf{C}}(\underline{\mathbf{P}}, \mathbf{x})$ for every $\underline{\mathbf{P}}$ in $\mathcal{N}(\underline{\mathbf{A}})$ with the associated sequences $\left\{\chi_{i}^{n}\right\}_{n=1}^{\infty}$ converging in $L^{\infty}$ weak * to $\theta_{i}$. This set is denoted by $\mathcal{G} \mathcal{A} d^{\infty}(K, \underline{\mathbf{A}}, \underline{\gamma})$. Similarly for a finite number of stress constraints the $\mathrm{G}$ closure of $\mathcal{A} d^{L}(K, \underline{\mathbf{A}}, \underline{\gamma})$ is denoted by $\mathcal{G} \mathcal{A} d^{L}(K, \underline{\mathbf{A}}, \underline{\gamma})$ and is defined to be all functions $\left(\widetilde{\mathbf{C}}(\underline{\mathbf{P}}, \mathbf{x}), \theta_{1}(\mathbf{x}), \ldots, \bar{\theta}_{N}(\mathbf{x})\right)$ for which there exists sequences $\left\{\mathbf{C}^{n}(\underline{\mathbf{P}}, \mathbf{x})\right\}_{i=1}^{n}$ in $\mathcal{A} d^{L}(K, \underline{\mathbf{A}}, \underline{\gamma}) \mathrm{G}$ converging to $\widetilde{\mathbf{C}}(\underline{\mathbf{P}}, \mathbf{x})$ for every $\underline{\mathbf{P}}$ in $\mathcal{N}(\underline{\mathbf{A}})$ with the associated sequences $\left\{\chi_{i}^{n}\right\}_{n=1}^{\infty}$ converging in $L^{\infty}$ weak $*$ to $\theta_{i}$. The sets $\mathcal{G} \mathcal{A} d^{\infty}(K, \underline{\mathbf{A}}, \underline{\gamma})$ and $\mathcal{G} \mathcal{A} d^{L}(K, \underline{\mathbf{A}}, \underline{\gamma})$ are referred to as stress constrained $\mathrm{G}$ closures of the original set of 
controls $\mathbf{C}(\underline{\mathbf{A}}, \mathbf{x})$ defined in the introduction. The relaxed formulations for $P^{\infty}$ and $P^{L}$ are given by

$$
\begin{aligned}
R P^{\infty}=\inf \left\{F(\mathbf{u}) ; \mathbf{u} \text { in } W_{0}^{1,2}\left(\Omega, \mathbf{R}^{3}\right),-\operatorname{div}(\widetilde{\widetilde{C}}(\underline{\mathbf{A}}, \mathbf{x}) \epsilon(\mathbf{u}))=\right. & \mathbf{f}, \widetilde{\mathbf{C}}(\underline{\mathbf{A}}, \mathbf{x}) \\
& \text { in } \left.\mathcal{G} \mathcal{A} d^{\infty}(K, \underline{\mathbf{A}}, \underline{\gamma})\right\}
\end{aligned}
$$

and

$$
\begin{aligned}
R P^{L}=\inf \left\{F(\mathbf{u}) ; \mathbf{u} \text { in } W_{0}^{1,2}\left(\Omega, \mathbf{R}^{3}\right),-\operatorname{div}(\widetilde{\mathbf{C}}(\underline{\mathbf{A}}, \mathbf{x}) \epsilon(\mathbf{u}))=\right. & \mathbf{f}, \widetilde{\mathbf{C}}(\underline{\mathbf{A}}, \mathbf{x}) \\
& \text { in } \left.\mathcal{G} \mathcal{A} d^{L}(K, \underline{\mathbf{A}}, \underline{\gamma})\right\} .
\end{aligned}
$$

The desired properties of the relaxed problems are given in the following theorem.

ThEOREM 4.1. The relaxed problems have the following properties.

1. $R P^{\infty}=P^{\infty}$ and $R P^{L}=P^{L}$.

2. There exists a function $\widehat{\mathbf{C}}(\underline{\mathbf{P}}, \mathbf{x})$ in $\mathcal{G} \mathcal{A} d^{\infty}(K, \underline{\mathbf{A}}, \underline{\gamma})$ and displacement $\hat{\mathbf{u}}$ in $W_{0}^{1,2}\left(\Omega, \mathbf{R}^{3}\right)$ for which

$$
-\operatorname{div}(\widehat{\mathbf{C}}(\underline{\mathbf{A}}, \mathbf{x}) \epsilon(\hat{\mathbf{u}}))=\mathbf{f},
$$

and

$$
R P^{\infty}=F(\hat{\mathbf{u}}) .
$$

Similarly there exists a function $\mathbf{C}^{*}(\underline{\mathbf{P}}, \mathbf{x})$ in $\mathcal{G} \mathcal{A} d^{L}(K, \underline{\mathbf{A}}, \underline{\gamma})$ and displacement $\mathbf{u}^{*}$ in $W_{0}^{1,2}\left(\Omega, \mathbf{R}^{3}\right)$ for which

$$
-\operatorname{div}\left(\mathbf{C}^{*}(\underline{\mathbf{A}}, \mathbf{x}) \epsilon\left(\mathbf{u}^{*}\right)\right)=\mathbf{f},
$$

and

$$
R P^{L}=F\left(\mathbf{u}^{*}\right)
$$

3. There exists a recovery sequence of controls $\left\{\mathbf{C}^{n}\right\}_{n=1}^{\infty}$ in $\mathcal{A} d^{\infty}(K, \underline{\mathbf{A}}, \underline{\gamma}) \mathrm{G}$ converging to $\widehat{\mathbf{C}}(\underline{\mathbf{A}}, \mathbf{x})$. Similarly there exists a recovery sequence of controls $\left\{\mathbf{C}^{n}\right\}_{n=1}^{\infty}$ in $\mathcal{A} d^{L}(K, \underline{\mathbf{A}}, \underline{\gamma}) \mathrm{G}$ converging to $C^{*}(\underline{\mathbf{A}}, \mathbf{x})$.

As it stands, the definition of the stress constrained $G$ closure is given in terms of constraints on $\mathrm{G}$ convergent sequences of controls. We show that the explicit characterization of $\mathcal{G} \mathcal{A} d^{L}(K, \underline{\mathbf{A}}, \underline{\gamma})$ can be found and is given in terms of constraints on suitable homogenized quantities. For the set $\mathcal{G} \mathcal{A} d^{\infty}(K, \underline{\mathbf{A}}, \underline{\gamma})$ we develop an upper bound in terms of homogenized quantities.

In order to characterize $\mathcal{G} \mathcal{A} d^{L}(K, \underline{\mathbf{A}}, \underline{\gamma})$ we introduce a new set of controls. We begin with the set $\mathcal{C}(\mathbf{A}, \underline{\gamma})$ given by all controls $\mathbf{C}(\underline{\mathbf{P}}, \mathbf{x})=\sum_{i=1}^{N} \mathbf{P}_{i} \chi_{i}(\mathbf{x})$ with $\underline{\mathbf{P}}$ in $\mathcal{N}(\underline{\mathbf{A}}), \int_{\Omega} \chi_{i} d \mathbf{x} \leq \gamma_{i}$. Next we consider the unconstrained G closure of $\mathcal{C}(\mathbf{A}, \underline{\gamma})$ G converging to $\widetilde{\mathbf{C}}(\underline{\mathbf{P}}, \mathbf{x})$ for every $\underline{\mathbf{P}}$ in $\mathcal{N}(\underline{\mathbf{A}})$ with the associated sequences $\left\{\chi_{i}^{n}\right\}_{n=1}^{\infty}$ converging in $L^{\infty}$ weak $*$ to $\theta_{i}$. This set is denoted by $\mathcal{G C}(\mathbf{A}, \underline{\gamma})$. The set $\mathcal{H}^{L}(K, \mathbf{A}, \underline{\gamma})$ is defined to be all $\widetilde{\mathbf{C}}(\underline{\mathbf{P}}, \mathbf{x})$ in $\mathcal{G C}(\mathbf{A}, \underline{\gamma})$ for which the associated stress is given by $\sigma=\widetilde{\mathbf{C}}(\underline{\mathbf{A}}, \mathbf{x}) \epsilon(\mathbf{v})$, where $\mathbf{v}$ is the $W_{0}^{1,2}\left(\Omega, \overline{\mathbf{R}^{3}}\right)$ solution of

$$
-\operatorname{div}(\widetilde{\mathbf{C}}(\underline{\mathbf{A}}, \mathbf{x}) \epsilon(\mathbf{v}))=\mathbf{f},
$$


and $\sigma$ satisfies

$$
\int_{\Omega} p_{j}(\mathbf{x})(\mathbf{I}+\widetilde{\mathbf{Q}}(\mathbf{x})) \sigma(\mathbf{x}): \sigma(\mathbf{x}) d \mathbf{x} \leq K^{2} \int_{\Omega} p_{j}(\mathbf{x}) d \mathbf{x}, \text { for } j=1, \ldots, L,
$$

with

$$
\widetilde{\mathbf{Q}}(\mathbf{x})=\left\{\sum_{i=1}^{N}\left(\widetilde{\mathbf{S}}(\underline{\mathbf{A}}, \mathbf{x}) \mathbf{A}_{i}^{2} \vec{\nabla}^{i} \widetilde{\mathbf{C}}(\underline{\mathbf{A}}, \mathbf{x}) \widetilde{\widetilde{\mathbf{S}}}(\underline{\mathbf{A}}, \mathbf{x})\right\}-\mathbf{I},\right.
$$

and $\widetilde{\mathbf{S}}(\underline{\mathbf{A}}, \mathbf{x})=(\widetilde{\mathbf{C}}(\underline{\mathbf{A}}, \mathbf{x}))^{-1}$. The characterization of $\mathcal{G} \mathcal{A} d^{L}(K, \underline{\mathbf{A}}, \underline{\gamma})$ is given by the following

Theorem 4.2. For any $t>0$, one has

$$
\mathcal{H}^{L}(K-t, \mathbf{A}, \underline{\gamma}) \subset \mathcal{G} \mathcal{A} d^{L}(K, \underline{\mathbf{A}}, \underline{\gamma}) \subset \mathcal{H}^{L}(K, \mathbf{A}, \underline{\gamma})
$$

One can use the explicit set of controls provided by the class $\mathcal{H}^{L}(K, \mathbf{A}, \underline{\gamma})$ to provide a relaxed design problem given in terms of explicit homogenized quantities. We introduce the homogenized design problem

$$
H P^{L}=\inf _{\mathcal{H}^{L}(K, \underline{\mathbf{A}}, \gamma)} F(\mathbf{u}) .
$$

The first feature of the homogenized design problem is that the infimum $H P^{L}$ is attained by a function in $\mathcal{H}^{L}(K, \underline{\mathbf{A}}, \gamma)$, i.e.,

Theorem 4.3. There exists a function $\widehat{\mathbf{C}}(\underline{\mathbf{P}}, \mathbf{x})$ in $\mathcal{H}^{L}(K, \underline{\mathbf{A}}, \gamma)$ and displacement $\hat{\mathbf{u}}$ in $W_{0}^{1,2}\left(\Omega, \mathbf{R}^{3}\right)$ for which

$$
\begin{gathered}
-\operatorname{div}(\widehat{\widehat{\mathbf{C}}}(\underline{\mathbf{A}}, \mathbf{x}) \epsilon(\hat{\mathbf{u}}))=\mathbf{f}, \\
H P^{L}=F(\hat{\mathbf{u}}),
\end{gathered}
$$

where

$$
\int_{\Omega} p_{j}(\mathbf{x})(\mathbf{I}+\widehat{\mathbf{Q}}(\mathbf{x})) \hat{\sigma}(\mathbf{x}): \hat{\sigma}(\mathbf{x}) d \mathbf{x} \leq K^{2} \int_{\Omega} p_{j}(\mathbf{x}) d \mathbf{x}, \text { for } j=1, \ldots, L,
$$

and

$$
\widehat{\mathbf{Q}}(\mathbf{x})=\left\{\sum_{i=1}^{N}\left(\widehat{\mathbf{S}}(\underline{\mathbf{A}}, \mathbf{x}) \mathbf{A}_{i}^{2} \vec{\nabla}^{i} \widehat{\mathbf{C}}(\underline{\mathbf{A}}, \mathbf{x}) \widehat{\mathbf{S}}(\underline{\mathbf{A}}, \mathbf{x})\right)\right\}-\mathbf{I} .
$$

The second important feature is the connection between the minimizer $\widehat{\mathbf{C}}(\underline{\mathbf{P}}, \mathbf{x})$ of the homogenized design problem and nearly optimal configurations. To make the connection the following optimal design problems are introduced.

$$
P_{k}^{L}=\inf _{\mathbf{C}(\underline{\mathbf{P}}, \mathbf{x}) \text { in } \mathcal{C}(\underline{\mathbf{A}}, \underline{\gamma})} F(\mathbf{u})
$$

subject to the constraints:

$$
\int_{\Omega} p_{j}(\mathbf{x})|\sigma|^{2} d \mathbf{x} \leq\left(1+\frac{1}{k}\right) K^{2} \int_{\Omega} p_{j}(\mathbf{x}) d \mathbf{x}, j=1, \ldots, L,
$$

where $\mathbf{u}$ is the $W_{0}^{1,2}\left(\Omega, \mathbf{R}^{3}\right)$ solution to the state equation

$$
-\operatorname{div}(\mathbf{C}(\underline{\mathbf{A}}, \mathbf{x}) \epsilon(\mathbf{u}))=\mathbf{f}
$$


and $\sigma=\mathbf{C}(\underline{\mathbf{A}}, \mathbf{x}) \epsilon(\mathbf{u})$. It is clear that as $k$ tends to infinity, the constraints given in the design problems $P_{k}^{L}$ approach the stress constraints associated with $P^{L}$. The problems $P_{k}^{L}$ share the same feature as the problem $P^{L}$ in that they are optimal design problems over admissible configurations of $N$ linear elastic materials. The connection between the minimizer of $H P^{L}$ and nearly optimal configurations is given in the following theorem.

THEOREM 4.4. Given a minimizer $\widehat{\mathbf{C}}(\underline{\mathbf{P}}, \mathbf{x})$ of $H P^{L}$, then there is a sequence of configurations and associated controls $\left\{\mathbf{C}^{k}(\underline{\mathbf{P}}, \mathbf{x})\right\}_{k=1}^{\infty}$ in $\mathcal{C}(\underline{\mathbf{A}}, \underline{\gamma})$ such that for all $\underline{\mathbf{P}}$ in $\mathcal{N}(\underline{\mathbf{A}})$, the sequence $\left\{\mathbf{C}^{k}(\underline{\mathbf{P}}, \mathbf{x})\right\}_{k=1}^{\infty}$ G-converges to $\widehat{\mathbf{C}}(\underline{\mathbf{P}}, \mathbf{x})$ and the stresses $\sigma^{k}$ satisfy the constraints (4.21). For this case, one has

$$
\lim _{k \rightarrow \infty} F\left(\mathbf{u}^{k}\right)=H P^{L} .
$$

Moreover, given any $\varepsilon>0$, there exists an index $J>0$ such that for all $k>J$,

$$
P_{k}^{L} \leq F\left(\mathbf{u}^{k}\right) \leq P_{k}^{L}+\varepsilon
$$

and

$$
\lim _{k \rightarrow \infty} P_{k}^{L}=H P^{L} .
$$

Thus one can use the minimizer for the homogenized design problem to recover nearly optimal stress constrained designs. It is clear that the homogenized design problem introduced here provides a way to identify nearly optimal configurations to a family of stress constrained problems with constraints approaching the original problem. Indeed, if instead of considering the problem $P^{L}$ we consider a problem with the stress constraints given in terms of a slightly larger tolerance $K^{2}(1+1 / k)$, the homogenized problem delivers an admissible and nearly optimal configuration.

Next, an upper bound for the set $\mathcal{G} \mathcal{A} d^{\infty}(K, \underline{\mathbf{A}}, \underline{\gamma})$ is provided. The set $\mathcal{H}^{\infty}(K, \mathbf{A}, \underline{\gamma})$ is defined to be all $\widetilde{\mathbf{C}}(\underline{\mathbf{P}}, \mathbf{x})$ in $\mathcal{G C}(\mathbf{A}, \underline{\gamma})$ for which the associated stress is given by $\sigma=\widetilde{\mathbf{C}}(\underline{\mathbf{A}}, \mathbf{x}) \epsilon(\mathbf{v})$, where $\mathbf{v}$ is the $W_{0}^{1,2}\left(\Omega, \mathbf{R}^{3}\right)$ solution of

$$
-\operatorname{div}(\widetilde{\mathbf{C}}(\underline{\mathbf{A}}, \mathbf{x}) \epsilon(\mathbf{v}))=\mathbf{f}
$$

and $\sigma$ satisfies

$$
(\mathbf{I}+\widetilde{\mathbf{Q}}(\mathbf{x})) \sigma(\mathbf{x}): \sigma(\mathbf{x}) \leq K^{2}
$$

almost everywhere. The upper bound for the set $\mathcal{G} \mathcal{A} d^{\infty}(K, \underline{\mathbf{A}}, \underline{\gamma})$ is given by

THEOREM 4.5.

$$
\mathcal{G} \mathcal{A} d^{\infty}(K, \underline{\mathbf{A}}, \underline{\gamma}) \subset \mathcal{H}^{\infty}(K, \mathbf{A}, \underline{\gamma}) .
$$

As before, one can use the explicit set of controls provided by the class $\mathcal{H}^{\infty}(K, \mathbf{A}, \underline{\gamma})$ to identify nearly optimal configurations for stress constrained problems. We introduce the homogenized design problem

$$
H P^{\infty}=\inf _{\mathcal{H} \infty(K, \underline{\mathbf{A}}, \gamma)} F(\mathbf{u}) .
$$

The first feature of the homogenized design problem is that the infimum $H P^{\infty}$ is attained by a function in $\mathcal{H}^{\infty}(K, \underline{\mathbf{A}}, \gamma)$, i.e., 
TheOREM 4.6. There exists a function $\widehat{\mathbf{C}}(\underline{\mathbf{P}}, \mathbf{x})$ in $\mathcal{H}^{\infty}(K, \underline{\mathbf{A}}, \gamma)$ and displacement $\hat{\mathbf{u}}$ in $W_{0}^{1,2}\left(\Omega, \mathbf{R}^{3}\right)$ for which

$$
-\operatorname{div}(\widehat{\mathbf{C}}(\underline{\mathbf{A}}, \mathbf{x}) \epsilon(\hat{\mathbf{u}}))=\mathbf{f}
$$

and

$$
H P^{\infty}=F(\hat{\mathbf{u}})
$$

where

$$
(\mathbf{I}+\widehat{\mathbf{Q}}(\mathbf{x})) \hat{\sigma}(\mathbf{x}): \hat{\sigma}(\mathbf{x}) \leq K^{2}
$$

almost everywhere.

Similarly, there is a connection between the minimizer $\widehat{\mathbf{C}}(\underline{\mathbf{P}}, \mathbf{x})$ of the homogenized design problem $H P^{\infty}$ and nearly optimal configurations. To make the connection consider a countable dense subset $\left\{p_{\ell}\right\}_{\ell=1}^{\infty}$ of the nonnegative functions in $C^{\infty}(\bar{\Omega})$. For a finite collection of these functions $S_{M I}=\left\{p_{\ell}\right\}_{\ell=1}^{M I}$, the following optimal design problems are introduced:

$$
\widetilde{P}^{M}=\inf _{\mathbf{C}(\underline{\mathbf{P}}, \mathbf{x}) \text { in } \mathcal{C}(\underline{\mathbf{A}}, \underline{\gamma})} F(\mathbf{u}),
$$

subject to the constraints:

$$
\int_{\Omega} p_{\ell}(\mathbf{x})|\sigma|^{2} d \mathbf{x} \leq\left(1+\frac{1}{M}\right) K^{2} \int_{\Omega} p_{\ell}(\mathbf{x}) d \mathbf{x}, \text { for } p_{\ell} \text { in } S_{M}
$$

where $\mathbf{u}$ is the $W_{0}^{1,2}\left(\Omega, \mathbf{R}^{3}\right)$ solution to the state equation

$$
-\operatorname{div}(\mathbf{C}(\underline{\mathbf{A}}, \mathbf{x}) \epsilon(\mathbf{u}))=\mathbf{f}
$$

and $\sigma=\mathbf{C}(\underline{\mathbf{A}}, \mathbf{x}) \epsilon(\mathbf{u})$. It is clear that as $M$ tends to infinity, the constraints given in the design problems $\widetilde{P}^{M}$ approach the constraint given by (1.8). The problems $\widetilde{P}^{M}$ share the same feature as the problem $P^{\infty}$ in that they are optimal design problems over admissible configurations of $N$ linear elastic materials. The connection between the minimizer of $H P^{\infty}$ and nearly optimal configurations is given in the following theorem.

THEOREM 4.7. Given a minimizer $\widehat{\mathbf{C}}(\underline{\mathbf{P}}, \mathbf{x})$ of $H P^{\infty}$, then there is a sequence of configurations and associated controls $\left\{\mathbf{C}^{M}(\underline{\mathbf{P}}, \mathbf{x})\right\}_{M=1}^{\infty}$ in $\mathcal{C}(\underline{\mathbf{A}}, \underline{\gamma})$ such that for all $\underline{\mathbf{P}}$ in $\mathcal{N}(\underline{\mathbf{A}})$, the sequence $\left\{\mathbf{C}^{M}(\underline{\mathbf{P}}, \mathbf{x})\right\}_{M=1}^{\infty}$ G-converges to $\widehat{\mathbf{C}}(\underline{\mathbf{P}}, \mathbf{x})$ and the stresses $\sigma^{M I}$ satisfy the constraints (4.34). For this case, one has

$$
\lim _{M \rightarrow \infty} F\left(\mathbf{u}^{M}\right)=H P^{\infty} .
$$

Moreover, given any $\varepsilon>0$, there exists an index $J>0$ such that for all $M>J$,

$$
\widetilde{P}^{M} \leq F\left(\mathbf{u}^{M}\right) \leq \widetilde{P}^{M I}+\varepsilon
$$

and

$$
\lim _{M \rightarrow \infty} \widetilde{P}^{M}=H P^{\infty} .
$$


4.1. Proofs of Theorems 4.1, 4.2, and 4.5. The proof of Theorem 4.1 is given for the problem $R P^{\infty}$, noting that the properties of $R P^{L}$ are established in the same way. The inequality $R P^{\infty} \leq P^{\infty}$ follows, noting that $\mathcal{A} d^{\infty}(K, \underline{\mathbf{A}}, \underline{\gamma}) \subset \mathcal{G} \mathcal{A} d^{\infty}(K, \underline{\mathbf{A}}, \underline{\gamma})$. A special sequence of controls with elastic tensors in $\mathcal{A} d^{\infty}(K, \underline{\mathbf{A}}, \underline{\gamma})$ is constructed. Consider a minimizing sequence $\left\{\mathbf{C}^{E, n}(\underline{\mathbf{A}}, \mathbf{x})\right\}_{n=1}^{\infty}$ in $\mathcal{G} \mathcal{A} d^{\infty}(K, \underline{\mathbf{A}}, \underline{\gamma})$ for the problem $R P^{\infty}$. The associated sequence of elastic displacements is denoted by $\left\{\mathbf{u}^{n}\right\}_{n=1}^{\infty}$ and $R P^{\infty}=$ $\lim _{n \rightarrow \infty} F\left(\mathbf{u}^{n}\right)$. For each $n$ there exists a sequence of elastic tensors $\left\{\mathbf{C}^{n, k}(\underline{\mathbf{A}}, \mathbf{x})\right\}_{k=1}^{\infty}$ in $\mathcal{A} d^{\infty}(K, \underline{\mathbf{A}}, \underline{\gamma})$ for which $\mathbf{C}^{n, k}(\underline{\mathbf{A}}, \mathbf{x}), \mathrm{G}$ converges to $\mathbf{C}^{E, n}(\underline{\mathbf{A}}, \mathbf{x})$. The associated displacements $\mathbf{u}^{n, \bar{k}}$ in $W_{0}^{1,2}\left(\Omega ; \mathbf{R}^{3}\right)$ satisfy the state equation

$$
-\operatorname{div}\left(\mathbf{C}^{n, k}(\underline{\mathbf{A}}, \mathbf{x}) \epsilon\left(\mathbf{u}^{n, k}\right)\right)=f .
$$

For a given $n$ there exists an index $k_{n}$ and elastic tensor in $\mathcal{A} d^{\infty}(K, \underline{\mathbf{A}}, \underline{\gamma})$ such that

$$
\left|F\left(\mathbf{u}^{n, k_{n}}\right)-F\left(\mathbf{u}^{n}\right)\right|<1 / n
$$

and

$$
-\operatorname{div}\left(\mathbf{C}^{n, k_{n}}(\underline{\mathbf{A}}, \mathbf{x}) \epsilon\left(\mathbf{u}^{n, k_{n}}\right)\right)=\mathbf{f} .
$$

Passing to a subsequence if necessary and appealing to the fundamental property of $\mathrm{G}$ convergence given in Sec. 2, there exists a function $\widetilde{\mathbf{C}}^{E}(\underline{\mathbf{A}}, \mathbf{x})$ in $\mathcal{G} \mathcal{A} d^{\infty}(K, \underline{\mathbf{A}}, \underline{\gamma})$ for which

$$
\left\{\mathbf{C}^{n, k_{n}}(\underline{\mathbf{A}}, \mathbf{x})\right\}_{n=1}^{\infty}, \text { G converges to } \widetilde{\mathbf{C}}^{E}(\underline{\mathbf{A}}, \mathbf{x}) \text {. }
$$

The displacement associated with the effective elasticity $\widetilde{\mathbf{C}}^{E}(\underline{\mathbf{A}}, \mathbf{x})$ is denoted by $\tilde{\mathbf{u}}$ and

$$
-\operatorname{div}\left(\widetilde{\mathbf{C}}^{E}(\underline{\mathbf{A}}, \mathbf{x}) \epsilon(\tilde{\mathbf{u}})\right)=\mathbf{f} .
$$

Properties 1, 2, and 3 of Theorem 4.1 follow immediately, noting that the objective function $F(\cdot)$ is continuous with respect to $\mathrm{G}$ convergence.

To prove Theorem 4.2 , we start by showing that $\mathcal{H}^{L}(K-t, \mathbf{A}, \underline{\gamma}) \subset \mathcal{G} \mathcal{A} d^{L}(K, \underline{\mathbf{A}}, \underline{\gamma})$. Given $\widetilde{\mathbf{C}}(\underline{\mathbf{P}}, \mathbf{x})$ in $\mathcal{H}^{L}(K-t, \mathbf{A}, \underline{\gamma})$ there is a sequence $\left\{\mathbf{C}^{n}(\underline{\mathbf{P}}, \mathbf{x})\right\}_{n=1}^{\infty}$ in $\mathcal{C}(\mathbf{A}, \underline{\gamma}) \mathrm{G}$ converging to $\widetilde{\mathbf{C}}(\underline{\mathbf{P}}, \mathbf{x})$ for every $\underline{\mathbf{P}}$ in $\mathcal{N}(\underline{\mathbf{A}})$. The associated stresses are given by $\sigma^{n}=\mathbf{C}^{n}(\underline{\mathbf{A}}, \mathbf{x}) \epsilon\left(\mathbf{u}^{n}\right)$ and $\sigma=\widetilde{\mathbf{C}}(\underline{\mathbf{A}}, \mathbf{x}) \epsilon(\tilde{\mathbf{u}})$. Application of Theorem 3.1 gives

$$
\begin{aligned}
K^{2} \int_{\Omega} p_{j} d \mathbf{x} & >(K-t)^{2} \int_{\Omega} p_{j} d \mathbf{x} \\
& \geq \int_{\Omega} p_{j}(\widetilde{\mathbf{Q}}+\mathbf{I}) \sigma: \sigma d \mathbf{x} \\
& =\lim _{n \rightarrow \infty} \int_{\Omega} p_{j}\left|\sigma^{n}\right|^{2} d \mathbf{x} .
\end{aligned}
$$

From (4.44) it is evident that there exists an index $N$ such that $\left\{\mathbf{C}^{n}(\underline{\mathbf{P}}, \mathbf{x})\right\}_{n=N}^{\infty} \subset$ $\mathcal{A} d^{L}(K, \underline{\mathbf{A}}, \underline{\gamma})$; hence $\widetilde{\mathbf{C}}(\underline{\mathbf{P}}, \mathbf{x})$ is in $\mathcal{G} \mathcal{A} d^{L}(K, \underline{\mathbf{A}}, \underline{\gamma})$. Next we show $\mathcal{G} \mathcal{A} d^{L}(K, \underline{\mathbf{A}}, \underline{\gamma}) \subset$ $\mathcal{H}^{L}(K, \mathbf{A}, \underline{\gamma})$. Given $\widetilde{\mathbf{C}}(\underline{\mathbf{P}}, \mathbf{x})$ in $\mathcal{G} \mathcal{A} d^{L}(K, \underline{\mathbf{A}}, \underline{\gamma})$, there is a sequence $\left\{\mathbf{C}^{n}(\underline{\mathbf{P}}, \mathbf{x})\right\}_{n=1}^{\infty}$ in $\mathcal{A} d^{L}(K, \underline{\mathbf{A}}, \underline{\gamma})$ that $\mathrm{G}$ converges to $\widetilde{\mathbf{C}}(\underline{\mathbf{P}}, \mathbf{x})$. The associated stresses are given by $\sigma^{n}=$ 
$\mathbf{C}^{n}(\underline{\mathbf{A}}, \mathbf{x}) \epsilon\left(\mathbf{u}^{n}\right)$ and $\sigma=\widetilde{\mathbf{C}}(\underline{\mathbf{A}}, \mathbf{x}) \epsilon(\tilde{\mathbf{u}})$ and an application of Theorem 3.1 gives

$$
\begin{aligned}
K^{2} \int_{\Omega} p_{j} d \mathbf{x} & \geq \lim _{n \rightarrow \infty} \int_{\Omega} p_{j}\left|\sigma^{n}\right|^{2} d \mathbf{x} \\
& =\int_{\Omega} p_{j}(\widetilde{\mathbf{Q}}+\mathbf{I}) \sigma: \sigma d \mathbf{x}
\end{aligned}
$$

and $\widetilde{\mathbf{C}}(\underline{\mathbf{P}}, \mathbf{x})$ is an element of $\mathcal{H}^{L}(K, \mathbf{A}, \underline{\gamma})$.

Lastly, it is noted that Theorem 4.5 follows immediately from Theorem 3.2.

4.2. Proofs of the properties of the homogenized design problems. In this section the properties of the homogenized design problems given by Theorems 4.3, 4.4, 4.6, and 4.7 are established. Proofs of Theorems 4.6 and 4.7 are given noting that the proofs of Theorems 4.3 and 4.4 follow the same lines. A special sequence of elastic tensors in $\mathcal{C}(\underline{\mathbf{P}}, \gamma)$ is constructed. This sequence will be used to establish Theorems 4.6 and 4.7. To construct this sequence, consider a minimizing sequence $\left\{\mathbf{C}^{E, n}(\underline{\mathbf{P}}, \mathbf{x})\right\}_{n=1}^{\infty}$ for $H P^{\infty}$. The associated set of elastic displacements for the sequence $\left\{\mathbf{C}^{E, n}(\underline{\mathbf{A}}, \mathbf{x})\right\}_{n=1}^{\infty}$ is denoted by $\left\{\mathbf{u}^{n}\right\}_{n=1}^{\infty}$ and $H P^{\infty}=\lim _{n \rightarrow \infty} F\left(\mathbf{u}^{n}\right)$.

For each $n$ there exists a sequence of elasticity tensors $\left\{\mathbf{C}^{n, k}(\underline{\mathbf{P}}, \mathbf{x})\right\}_{k=1}^{\infty}$ in $\mathcal{C}(\underline{\mathbf{P}}, \underline{\gamma})$ such that $\mathbf{C}^{n, k}(\underline{\mathbf{P}}, \mathbf{x}), \mathrm{G}$ converges to $\mathbf{C}^{E, n}(\underline{\mathbf{P}}, \mathbf{x})$ for every $\underline{\mathbf{P}}$ in $\mathcal{N}(\underline{\mathbf{A}})$. The associated displacements $\mathbf{u}^{n, k}$ in $W_{0}^{1,2}\left(\Omega ; \mathbf{R}^{3}\right)$ satisfy the state equation

$$
-\operatorname{div}\left(\mathbf{C}^{n, k}(\underline{\mathbf{A}}, \mathbf{x}) \epsilon\left(\mathbf{u}^{n, k}\right)\right)=\mathbf{f}
$$

and we set $\sigma^{n, k}=\mathbf{C}^{n, k}(\underline{\mathbf{A}}, \mathbf{x}) \epsilon\left(\mathbf{u}^{n, k}\right)$. For every nonnegative differentiable function $p$, the constraint (4.27) together with Theorem 3.1 gives

$$
\lim _{k \rightarrow \infty} \int_{\Omega} p\left|\sigma^{n, k}\right|^{2} d \mathbf{x}=\int_{\Omega} p\left(\mathbf{Q}^{n}+\mathbf{I}\right) \sigma^{n}: \sigma^{n} d \mathbf{x} \leq K^{2} \int_{\Omega} p d \mathbf{x},
$$

where $\sigma^{n}(x)=\mathbf{C}^{E, n}(\underline{\mathbf{A}}, \mathbf{x}) \epsilon\left(\mathbf{u}^{n}\right)$ and

$$
\mathbf{Q}^{n}+\mathbf{I}=\sum_{i=1}^{N} \mathbf{S}^{E, n}(\underline{\mathbf{A}}, \mathbf{x}) \mathbf{A}_{i}^{2} \vec{\nabla}^{i} \mathbf{C}^{E, n}(\underline{\mathbf{A}}, \mathbf{x}) \mathbf{S}^{E, n}(\underline{\mathbf{A}}, \mathbf{x}) .
$$

A countable dense subset $\left\{p_{\ell}\right\}_{\ell=1}^{\infty}$ of the set of nonnegative differentiable functions continuous on the closure of the structural domain is introduced. Given $n$, put $S_{n}=\left\{p_{\ell}\right\}_{\ell=1}^{n}$; then there exists an index $k_{n}$ for which

$$
\begin{gathered}
\int_{\Omega} p_{\ell}\left|\sigma^{n, k_{n}}\right|^{2} d \mathbf{x} \leq K^{2} \int_{\Omega} p_{\ell}(1+1 / n) d \mathbf{x}, \text { for every } p_{\ell} \text { in } S_{n} \\
\left|F\left(\mathbf{u}^{n, k_{n}}\right)-F\left(\mathbf{u}^{n}\right)\right|<1 / n,
\end{gathered}
$$

where $\mathbf{u}^{n, k_{n}}$ is the $W_{0}^{1,2}\left(\Omega ; \mathbf{R}^{3}\right)$ solution of

$$
-\operatorname{div}\left(\mathbf{C}^{n, k_{n}}(\underline{\mathbf{A}}, \mathbf{x}) \epsilon\left(\mathbf{u}^{n, k_{n}}\right)\right)=\mathbf{f}
$$

and $\sigma^{n, k_{n}}=\mathbf{C}^{n, k_{n}}(\underline{\mathbf{A}}, \mathbf{x}) \epsilon\left(\mathbf{u}^{n, k_{n}}\right)$. Passing to a subsequence if necessary and appealing to the fundamental property of $\mathrm{G}$ convergence given in Sec. 2, there exists a function $\widetilde{\mathbf{C}}^{E}(\underline{\mathbf{P}}, \mathbf{x})$ in $\mathcal{C}(\underline{\mathbf{A}}, \mathbf{x})$ for which

$$
\left\{\mathbf{C}^{n, k_{n}}(\underline{\mathbf{P}}, \mathbf{x})\right\}_{n=1}^{\infty}, \mathrm{G} \text { converges to } \widetilde{\mathbf{C}}^{E}(\underline{\mathbf{P}}, \mathbf{x})
$$


for all $\underline{\mathbf{P}}$ in $\mathcal{N}(\underline{\mathbf{A}})$. The sequence $\left\{\left(\mathbf{C}^{n, k_{n}}(\underline{\mathbf{A}}, \mathbf{x})\right\}_{n=1}^{\infty}\right.$ constructed above satisfying (4.494.52) is called a configuration minimizing sequence and it is evident that $\lim _{n \rightarrow \infty} F\left(\mathbf{u}^{n, k_{n}}\right)=H P^{\infty}$.

The displacement associated with the effective elasticity $\widetilde{\mathbf{C}}^{E}(\underline{\mathbf{A}}, \mathbf{x})$ is denoted by $\widetilde{\mathbf{u}}$, where

$\tilde{\sigma}=\widetilde{\mathbf{C}}^{E}(\underline{\mathbf{A}}, \mathbf{x}) \epsilon(\tilde{\mathbf{u}})$ and

$$
-\operatorname{div}\left(\widetilde{\mathbf{C}}^{E}(\underline{\mathbf{A}}, \mathbf{x}) \epsilon(\tilde{\mathbf{u}})\right)=\mathbf{f}
$$

$$
\widetilde{\mathbf{Q}}+\mathbf{I}=\sum_{i=1}^{N} \widetilde{\mathbf{S}}^{E}(\underline{\mathbf{A}}, \mathbf{x}) \mathbf{A}_{i}^{2} \nabla^{i} \widetilde{\mathbf{C}}^{E}(\underline{\mathbf{A}}, \mathbf{x}) \widetilde{\mathbf{S}}^{E} .
$$

We proceed to establish Theorem 4.6. For each $p_{\ell}$ in $\left\{p_{\ell}\right\}_{\ell=1}^{\infty}$, it follows that

$$
\begin{aligned}
\int_{\Omega} p_{\ell}(\widetilde{\mathbf{Q}}+\mathbf{I}) \tilde{\sigma}: \tilde{\sigma} d \mathbf{x} & =\lim _{n \rightarrow \infty} \int_{\Omega} p_{\ell}\left|\sigma^{n, k_{n}}\right|^{2} d \mathbf{x} \\
& \leq K^{2} \int_{\Omega} p_{\ell} d \mathbf{x} .
\end{aligned}
$$

Thus by density of $\left\{p_{\ell}\right\}_{\ell=1}^{\infty}$, it is evident that $\widetilde{\mathbf{C}}^{E}(\underline{\mathbf{P}}, \mathbf{x})$ is in $\mathcal{H}^{\infty}(K, \underline{\mathbf{A}}, \underline{\gamma})$. Theorem 4.6 follows from the continuity of $F(\cdot)$ and (4.50).

To establish Theorem 4.7, one considers the design problems $\widetilde{P}^{M}$ given by (4.33). It is first shown that $\widetilde{P}^{M} \leq H P^{\infty}$. Consider the configuration minimizing sequence $\left\{\left(\mathbf{C}^{n, k_{n}}(\underline{\mathbf{P}}, \mathbf{x})\right\}_{n=1}^{\infty}\right.$. It is evident that given the index $M$, one has that for all $n>M$, that $\mathbf{C}^{n, k_{n}}(\underline{\mathbf{A}}, \mathbf{x})$ is admissible for $\widetilde{P}^{M}$, and $\widetilde{P}^{M} \leq F\left(\mathbf{u}^{n, k_{n}}\right)$. Sending $n$ to infinity shows that $\widetilde{P}^{M} \leq H P^{\infty}$. Noting that $\widetilde{P}^{M}$ is monotone increasing with $M$ and bounded above implies the existence of $\lim _{M \rightarrow \infty} \widetilde{P}^{M}$. Next it is shown that $H P^{\infty}=\lim _{M \rightarrow \infty} \widetilde{P}^{M}$. Given $M>0$, one can choose $\mathbf{C}^{M}(\underline{\mathbf{P}}, \mathbf{x})$ in $\mathcal{C}(\underline{\mathbf{A}}, \underline{\gamma})$ with associated displacement $\mathbf{u}^{M}$ in $W_{0}^{1,2}\left(\Omega ; \mathbf{R}^{3}\right)$ for which

$$
\int_{\Omega} p_{k}\left|\sigma^{M}\right|^{2} d \mathbf{x} \leq K^{2} \int_{\Omega} p_{k}(1+1 / M) d \mathbf{x}, \text { for } p_{k} \text { in } S_{M}
$$

and

$$
-\operatorname{div}\left(\mathbf{C}^{M}(\underline{\mathbf{A}}, \mathbf{x}) \epsilon\left(\mathbf{u}^{M}\right)\right)=\mathbf{f},
$$

where

$$
\widetilde{P}^{M} \leq F\left(\mathbf{u}^{M}\right) \leq \widetilde{P}^{M}+1 / M
$$

From the fundamental property of $\mathrm{G}$ convergence and passage to subsequences if necessary, there exists an effective elasticity $\overline{\mathbf{C}}^{E}(\underline{\mathbf{P}}, \mathbf{x})$ in $\mathcal{G C}(\underline{\mathbf{A}}, \underline{\gamma})$ such that $\left\{\mathbf{C}^{M}(\underline{\mathbf{P}}, \mathbf{x})\right\}_{M=1}^{\infty}$ G converges to $\overline{\mathbf{C}}^{E}(\underline{\mathbf{P}}, \mathbf{x})$ for all $\underline{\mathbf{P}}$ in $\mathcal{N}(\underline{\mathbf{A}})$. The associated displacement $\overline{\mathbf{u}}$ in $W_{0}^{1,2}\left(\Omega ; \mathbf{R}^{3}\right)$ solves

$$
-\operatorname{div}\left(\overline{\mathbf{C}}^{E}(\underline{\mathbf{A}}, \mathbf{x}) \epsilon(\overline{\mathbf{u}})\right)=\mathbf{f} .
$$

Here $\lim _{M \rightarrow \infty} F\left(\mathbf{u}^{M}\right)=F(\overline{\mathbf{u}})$ and arguing as above one sees that $\overline{\mathbf{C}}^{\mathbf{E}}(\underline{\mathbf{A}}, \mathbf{x})$ is in $\mathcal{H}^{\infty}(K, \underline{\mathbf{A}}, \underline{\gamma})$. Observing that $H P^{\infty} \leq F(\overline{\mathbf{u}})=\lim _{M \rightarrow \infty}, F\left(\mathbf{u}^{M}\right)$ together with (4.58) gives the set of inequalities

$$
H P^{\infty} \leq \lim _{M \rightarrow \infty} F\left(\mathbf{u}^{M}\right) \leq \lim _{M \rightarrow \infty} \widetilde{P}^{M} \leq H P^{\infty} .
$$


One concludes that $H P^{\infty}=\lim _{M \rightarrow \infty} \widetilde{P}^{M}=F(\overline{\mathbf{u}})$ and that $\overline{\mathbf{C}}^{E}(\underline{\mathbf{P}}, \mathbf{x})$ is a minimizer of $H P^{\infty}$ and Theorem 4.7 follows.

5. Acknowledgements. This research effort is sponsored by NSF through grant DMS-0072469 and by the Air Force Office of Scientific Research, Air Force Materiel Command USAF, under grant number F49620-99-1-0009. The U.S. Government is authorized to reproduce and distribute reprints for governmental purposes notwithstanding any copyright notation thereon. The views and conclusions herein are those of the authors and should not be interpreted as necessarily representing the official policies or endorsements, either expressed or implied, of the Air Force Office of Scientific Research or the U.S. Government.

\section{REFERENCES}

[1] M. P. Bendsoe, Optimization of Structural Topology, Shape, and Material, Springer-Verlag, Berlin, 1995

[2] E. Bonnetier and M. Vogelius, An elliptic regularity result for a composite medium with touching fibers of circular cross-section, SIAM J. Math. Anal. 31, 651-677 (2000)

[3] A. Bensoussan, J.-L. Lions, and G. Papanicolaou, Asymptotic Analysis for Periodic Structures, Studies in Mathematics and its Applications, 5, North-Holland, Amsterdam, 1978

[4] G. Butazzo, Semicontinuity, Relaxation and Integral Representation in the Calculus of Variations, Pitman Research Notes in Mathematics, London, Harlow, 1989

[5] E. Cabib and G. Dal Maso, On a Class of Optimum Problems in Structural Design, Journal of Optimization Theory and Applications 56, 39-65 (1989)

[6] A. Cherkaev, Variational Methods for Structural Optimization, Springer-Verlag, New York, 2000

[7] B. Dacorogna, Direct Methods in the Calculus of Variations, Springer-Verlag, New York, 1989

[8] G. Dal Maso, An Introduction to $\Gamma$-Convergence, Birkhäuser, Boston, 1993

[9] Y. Grabovsky, Optimal Design Problems for Two-Phase Conducting Composites with Weakly Discontinuous Objective Functionals, Advances in Applied Mathematics 27, 683-704 (2001)

[10] R. V. Kohn and G. Strang, Optimal Design and Relaxation of Variational Problems, Communications on Pure and Applied Mathematics, 34, Part I, pp. 113-137, Part II, pp. 139-182, Part III, pp. 357-377 (1986)

[11] Y. Y. Li and M. Vogelius, Gradient estimates for solutions to divergence form elliptic equations with discontinuous coefficients, Arch. Rational Mech. Anal. 153, 91-151 (2000)

[12] Y. Y. Li and L. Nirenberg, Estimates for elliptic systems from composite material, Communications in Pure and Applied Mathematics, to appear

[13] R. Lipton, Relaxation through homogenization for optimal design problems with gradient constraints, Journal of Optimization Theory and Applications 114, 27-54 (2002)

[14] R. Lipton and A. P. Velo, Optimal Design of Gradient Fields with Applications to Electrostatics, Nonlinear Partial Differential Equations and Their Applications, College de France Seminar Volume XIV, D. Cioranescu and J.-L. Lions, Eds., Studies in Mathematics and its Applications, 31, North Holland, Amsterdam, 2002, pp. 509-522

[15] K. A. Lurie, On the Optimal Distribution of the Resistivity Tensor of the Working Substance in a Magnetohydrodynamic Channel, Journal of Applied Mathematics and Mechanics 34, 255-274 (1970)

[16] K. A. Lurie, Applied Optimal Control Theory of Distributed Systems, Plenum Press, New York and London, 1993

[17] K. A. Lurie and A. V. Cherkaev, Effective Characteristics of Composite Materials and the Optimal Design of Structural Elements, Uspekhi Mekhaniki (Advances in Mechanics) 9, 3-81 (1986)

[18] N. G. Meyers and A. Elcrat, Some results on regularity of non-linear elliptic equations and quasiregular functions, Duke Math. J. 47, 121-136 (1975)

[19] F. Murat, Un Contre-Example pour le Problème du Contrôle dans les Coefficients, Comptes Rendus de l'Academie des Sciences, Paris Séries A 273, 708-711 (1971) 
[20] F. Murat and L. Tartar, Calcul des Variations et Homogénéisation, Les Méthodes de l'Homogénéisation: Théorie et Applications en Physique, D. Bergman et al., Eds., Collection de la Direction des Études et Recherches d'Electricité de France, 57, Eyrolles, Paris, 1985, pp. 319-369

[21] F. Murat and L. Tartar, H Convergence, Topics in the Mathematical Modelling of Composite Materials, Edited by A. V. Cherkaev and R. V. Kohn, Birkhäuser, Boston, 1997, pp. 21-43

[22] P. Pedregal, Constrained quasiconvexity and structural optimization, Arch. Ration. Mech. Anal. 154, 325-342 (2000)

[23] P. Pedregal, Fully Explicit Quasiconvexification of the Square of the Gradient of the State in Optimal Design, Electronic Research Announcements of the American Mathematical Society 7, 72-78 (2001)

[24] U. Raitums, On the local representation of G-closure, Arch. Rational Mech. Anal. 158, 213-234 (2001)

[25] U. Raitums, Lecture Notes on G-Convergence, Convexification and Optimal Control Problems for Elliptic Equations, University of Jyväskylä Department of Mathematics Lecture Notes 39, Jyväskylä, 1997

[26] G. I. N. Rozvany, N. Olhoff, K. T. Cheng, and J. E. Taylor, On the Solid Plate Paradox in Structural Optimization, Journal of Structural Mechanics 10, 1-32 (1982)

[27] S. Spagnolo, Convergence in Energy Operators. Proceedings of the Third Symposium on Numerical Solutions of Partial Differential Equations, Edited by B. Hubbard, (College Park, 1975), Academic Press, New York, 1976, pp. 469-498

[28] L. Simon, On G-convergence of elliptic operators, Indiana University Mathematics Journal 28, 587-594 (1979)

[29] L. Tartar, An introduction to the homogenization method in optimal design, Springer Lecture Notes in Mathematics, Vol. 1740, 2000, pp. 45-156

[30] L. Tartar, Remarks on Optimal Design Problems, Calculus of Variations, Homogenization and Continuum Mechanics, Edited by G. Buttazzo, G. Bouchitte, and P. Suquet, World Scientific, Singapore, 1994, pp. 279-296

[31] A. Velo, Optimal Design of Gradient Fields with Applications to Electrostatics, Ph.D. Thesis, Department of Mathematical Sciences, Worcester Polytechnic Institute, 2000

[32] V. V. Zhikov, S. M. Kozlov, and O. A. Oleinik, Homogenization of Differential Operators and Integral Functionals, Springer-Verlag, Berlin, 1994 